Mit eDemocracy zu deliberativer Politik?

Zur Praxis und Anschlussfähigkeit eines neuen Mediums

Hilmar Westholm 


\title{
Mit eDemocracy zu deliberativer Politik? Zur Praxis und Anschluss- fähigkeit eines neuen Mediums
}

\section{Hilmar Westholm}

Forschungsgruppe Telekommunikation der Universität Bremen, E-Mail: westholm@tzi.de

\section{Keywords}

Politische Partizipation, deliberative Politik, Bürgerbeteiligung, digitale Demokratie, eDemocracy, Anschlussfähigkeit, Internet, Informations- und Kommunikationstechnik

\begin{abstract}
Informations- und Kommunikationstechnik wird in zunehmendem Maße auch zur Unterstützung von Bürgerbeteiligung verwendet. Der Beitrag untersucht unter Bezugnahme auf das Modell deliberativer Politik beispielhaft an einer Online-Konsultation und anderen eDemocracy-Tools, ob der Einsatz neuer Technik demokratische Verfahren dahingehend verbessern kann, dass die Kommunikation unter den Beteiligten und damit der rationale Diskurs vor der Entscheidung positiv beeinflusst wird. Als wesentliche Voraussetzung für den Einsatz neuer Technik in Bürgerbeteiligungsprozessen wird der Begriff der „Anschlussfähigkeit“ eingeführt, wonach diese Techniken nicht nur aus technischem Blickwinkel beurteilt werden dürfen, sondern zudem unter rechtlichen, organisatorischen, ökonomischen, kulturellen und politischen Gesichtspunkten zu prüfen ist, ob sie in die vorgesehene Umgebung ,passen“.
\end{abstract}

( ) $\mathbf{T} \mathbf{A}$ - Institut für Technikfolgen-Abschätzung der Österreichischen Akademie der Wissenschaften A- 1030 Wien, Strohgasse 45/5, Fax: +43-1-710 9883 http://www.oeaw.ac.at/ita/ 


\section{Inhalt}

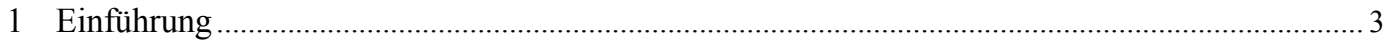

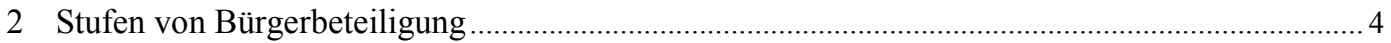

3 Qualität und Formen der Kommunikation am Beispiel einer Online-Konsultation....................... 6

3.1 Kennzeichen und Nutzungsmöglichkeiten der Online-Konsultation „Forum Horn-Lehe“................ 6

3.2 Beteiligung und Inhalt im Online-Forum Horn-Lehe .................................................................... 10

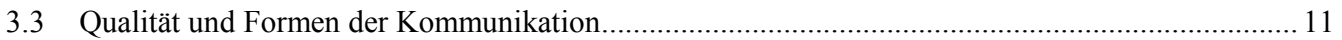

3.4 Erfahrungen mit Online-Beteiligung am Beispiel der Bauleitplanung ......................................... 14

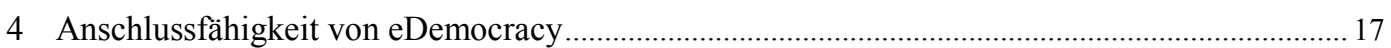

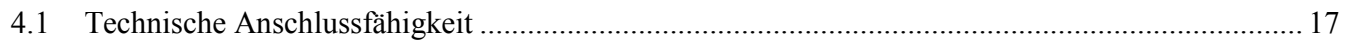

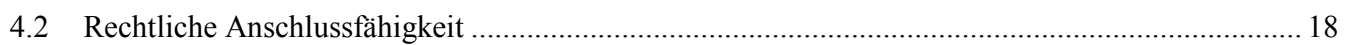

4.3 Einbettung in bestehende Organisationszusammenhänge ............................................................... 19

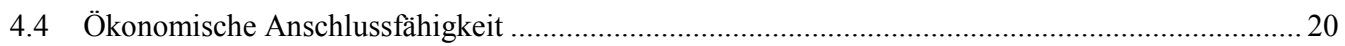

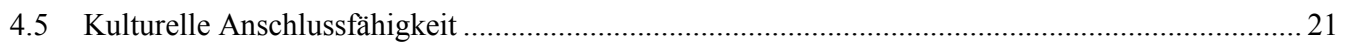

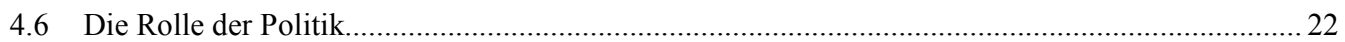

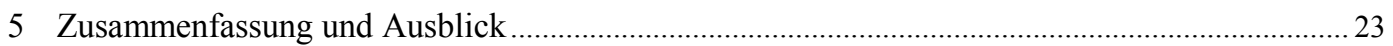

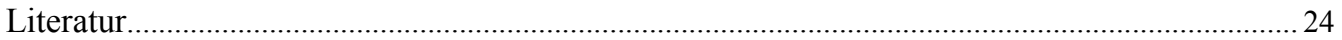

Dieses ITA-manu:script ist die überarbeitete Fassung eines Vortrags, den der Verfasser auf der Zweiten österr. TA-Konferenz TA'02 am 27. Mai 2002 gehalten hat. 


\section{Einführung}

Von neuen Medien wurde immer schon erwartet, die Demokratie zu stärken; für das Internet gilt dies unter dem Stichwort eDemocracy in ganz besonderem Maße. In der Diskussion um digitale oder elektronische Demokratie werden zumeist elektronische Kommunikationsformen subsumiert, die traditionelle - formelle wie informelle - Partizipationsverfahren unterstützen (beispielsweise Planauslegungen oder Diskussionen im Internet, Anfragen per Email, Kampagnen, Lobbyarbeit) oder ergänzen (virtuelle Parteitage, Online-Proteste wie Sit-Ins, Online-Polling). Nur selten wird auch die grundlegende Veränderung von Verfahren durch IuK-Technologien impliziert (Lenk 1999) oder gar die Substitution bestimmter traditioneller Verfahrensbestandteile vorgeschlagen. Der diesem Aufsatz zugrunde liegende Begriff elektronischer Demokratie umfasst alle vier genannten Aspekte.

Der zweite Teil der Fragestellung greift eine demokratietheoretische Diskussion auf, in der - initiiert von Jürgen Habermas - dem liberalen Demokratiemodell einerseits und dem republikanischen andererseits ein drittes - diskurstheoretisch fundiertes - Verständnis von „deliberativer Politik“ zur Seite gestellt wird, mit dem beide zuerst genannten Modelle „,im Begriff einer idealen Prozedur für Beratung und Beschlussfassung" integriert werden, indem über die Ausgestaltung von demokratischen Verfahren ein „Zusammenhang zwischen Verhandlungen, Selbstverständigungs- und Gerechtigkeitsdiskursen" (Habermas 1997: 285f., Hervorhebg. im Orig.) hergestellt wird, um somit vernünftige bzw. faire Ergebnisse zu erzielen (von lat. deliberare: überlegen, beraten; [nach angestellter Überlegung] sich entscheiden). Auf diese Weise werde mit einer „höherstufigen Intersubjektivität von Verständigungsprozessen (gerechnet), die sich einerseits in der institutionalisierten Form von Beratungen in parlamentarischen Körperschaften sowie andererseits im Kommunikationsnetz politischer Öffentlichkeiten vollziehen“ (ebd.: 288). Gerade mit der Hinzuziehung der „politischen Öffentlichkeiten“ integriert Habermas die Zivilgesellschaft als dritten Bestandteil neben öffentlicher Administration (Staat) und ökonomischem Handlungssystem (Markt).

Elektronische Demokratie könnte nun, so die dem Beitrag zugrunde liegende Ausgangsthese, aufgrund ihrer unbegrenzten zeitlichen und räumlichen Verfügbarkeit und durch Nutzung des kostengünstigen Internet dazu beitragen, der Verwirklichung einer deliberativen Politik einen großen Schritt näher zu kommen.

Die Praxis hat gezeigt, dass mit der Anwendung digitaler Verfahren doch erhebliche Probleme verbunden sind, die stichwortartig mit „digitaler Spaltung“, „Anonymität“, „fehlender Repräsentativität", „begrenzter Informationsverarbeitungskapazität" und „Kosten“ umschrieben werden können. Dahinter steht die grundsätzliche Problematik, den technischen Aspekt der Innovation in den Mittelpunkt des Interesses zu rücken, daneben aber die „Anschlussfähigkeit“ an organisatorische Verfahrensabläufe, kulturelle, rechtliche, ökonomische Bedingungen, die alle wiederum eingebettet sind in ein politisches Umfeld, zu ignorieren. Der Beitrag illustriert diese Anschlussfähigkeit einerseits an Beispielen, die vom Autoren selbst durchgeführt wurden, andererseits durch Rückgriff auf in Deutschland, aber auch in anderen europäischen Staaten gemachte Erfahrungen mit digitaler Demokratie, wobei das Akteursfeld dahingehend eingegrenzt wird, dass nur Verfahren auf kommunaler Ebene betrachtet werden, die zwischen politisch-administrativem System und Bürgerinnen und Bürgern stehen (also z. B. parteiinterne und Lobbyaktivitäten der assoziierten Akteure der Zivilgesellschaft ausgenommen werden). Besonderer Wert wird auf die Untersuchung der Qualität der neuartigen Kommunikationsprozesse gelegt, weil gerade das demokratische Modell deliberativer Politik stark von der Qualität des Diskurses unter den Beteiligten abhängt. Dieses exemplarische Vorgehen ermöglicht eine vertiefende Einsicht und Illustration des abstrakten Terminus „Anschlussfähigkeit“" anhand von konkreten Anwendungen. 


\section{Stufen von Bürgerbeteiligung}

Beteiligungsverfahren kann man unter dem Gesichtspunkt einteilen, in welchem Maße die Kommunikation zwischen politisch-administrativem System und Bürgern ${ }^{1}$ auf letztere hin orientiert ist, und kommt dann zur Dreiteilung Information - Konsultation - aktive Beteiligung (vgl. OECD 2001), wobei man Wahlen und Bürgerentscheide als Verfahren mit höchster Verbindlichkeit noch ergänzen sollte (vgl. Tab. 1).

Tabelle 1: Stufen von politischer Partizipation

\begin{tabular}{|c|c|c|}
\hline „Offline”-Anwendungen & Typen & Online-Tools \\
\hline \multicolumn{3}{|c|}{$\begin{array}{c}\text { Information } \\
\text { (Kommunikation zwischen Politik/Verwaltung und Bürgern ist einseitig) }\end{array}$} \\
\hline $\begin{array}{l}\text { Informationsveranstaltung, PR, } \\
\text { Folder, Modelle, ,amtliche Bekannt- } \\
\text { machungen“; Presse(-konferenz), } \\
\text { Auslegung von Bebauungsplänen } \\
\text { zu Amtsöffnungszeiten }\end{array}$ & $\begin{array}{l}\text { Unterlagen der Fachplanung } \\
\text { in Kommunen; } \\
\text { Satzungsvorhaben, } \\
\text { Informationen über } \\
\text { Bürgerbegehren }\end{array}$ & $\begin{array}{l}\text { Verwaltungsportale (,,24/7“), } \\
\text { 3-D-Modelle, Pläne, } \\
\text { Abstimmungsalter-nativen im } \\
\text { Netz }\end{array}$ \\
\hline \multicolumn{3}{|c|}{$\begin{array}{c}\text { Konsultation } \\
\text { (Kommunikation zwischen Politik/Verwaltung und Bürgern ist gegenseitig) }\end{array}$} \\
\hline Briefe, Telefonate & $\begin{array}{l}\text { Anfragen, } \\
\text { Beschwerdemanagement }\end{array}$ & Email \\
\hline $\begin{array}{l}\text { Meinungsumfragen per Telefon oder } \\
\text { auf Straßen; Fokusgruppen }\end{array}$ & $\begin{array}{l}\text { Bürger- bzw. } \\
\text { Kundenbefragungen }\end{array}$ & Online-Polling \\
\hline $\begin{array}{l}\text { Wortmeldungen von Bürgern bei } \\
\text { Gremien manchmal zugelassen (zu- } \\
\text { meist nicht), Bürgerfragestunden des } \\
\text { Rates, Zukunftswerkstätten }\end{array}$ & „Anhörungen“ & $\begin{array}{l}\text { Diskussionsplattformen im } \\
\text { Netz, Chats }\end{array}$ \\
\hline $\begin{array}{l}\text { Anregungen bei Bürgerversammlungen } \\
\text { oder per Brief }\end{array}$ & $\begin{array}{l}\text { Fachplanungen } \\
\text { (z. B. Bauleitplanung) }\end{array}$ & $\begin{array}{l}\text { Anregungen per Email oder in } \\
\text { (teil-) öffentlichen Online-Foren }\end{array}$ \\
\hline \multicolumn{3}{|c|}{$\begin{array}{c}\text { Aktive Beteiligung } \\
\text { (Kommunikation zwischen Politik/Verwaltung und Bürgern ist partnerschaftlich) }\end{array}$} \\
\hline $\begin{array}{l}\text { Moderation, Runder Tisch, } \\
\text { Mediation (in Veranstaltungsräumen } \\
\text { mit festen vorgegebenen Zeiten) }\end{array}$ & $\begin{array}{l}\text { „informelle“ Beteiligungs- } \\
\text { formen wie bei Verhand- } \\
\text { lungsprozessen }\end{array}$ & $\begin{array}{l}\text { Online-Mediation und Internet- } \\
\text { Arbeitsgruppen (Dokumenten- } \\
\text { bereitstellung, Diskussions- } \\
\text { und Pollingtools) }\end{array}$ \\
\hline \multicolumn{3}{|c|}{ (Wahlen) } \\
\hline $\begin{array}{l}\text { Wahlbezirke, Stimmabgabe im Amt } \\
\text { zu festgelegten Zeiten, Briefwahl }\end{array}$ & Bürgerbegehren, Wahlen & $\begin{array}{l}\text { Online-Wahlen und Online- } \\
\text { Bürgerbegehren }\end{array}$ \\
\hline
\end{tabular}

Eine besondere Stärke der Informations- und Kommunikationstechnik (IuK) liegt in der Unterstützung von Information der Bürgerschaft durch die Verwaltung: Das Internet ermöglicht über Verwaltungsportale einen Zugriff auf Informationen 24 Stunden am Tag und sieben Tage in der Woche $(, 24 / 7 “)$ - es ist nun nicht mehr nötig, Amtsstuben mit eingeschränkten Öffnungszeiten aufzusu-

1 Die deutsche Sprache unterschlägt aufgrund der Übereinstimmung von Gattungsbegriff und männlicher Sprachform oft die weibliche Seite. Auch wenn der Autor dies als einen Mangel begreift, werden im Text zumeist nicht die männliche und weibliche Form zusammen aufgeführt, da dies zu umständlich erscheint („,Bürgerinnen und Bürger“) oder ästhetisch sehr eigenwillig (,BürgerInnen“). Soweit möglich, werden neutrale Begriffe benutzt. 
chen; einmalige Informationsveranstaltungen können versäumt werden, wenn die Kommune Informationen über Straßenbauten, Sanierungsgebiete, Bebauungspläne (gute Beispiele: Düsseldorf, Bonn und Rhein-Sieg-Kreis, Osnabrück), anschauliche Modelle (z. B. auch in Form von 3D-Animationen) oder die Namen von Kommunalpolitikern (Beispiel: Tameside, Großbritannien) ins Netz stellt. In Nordjütland wurde vor den Kommunalwahlen 2001 versucht, Nichtwähler zurückzugewinnen, indem Politiker und ihre Programme im Internet dargestellt wurden, indem Bürger - insbesondere die Zielgruppe der Jugendlichen - mit Politikern in Chats diskutieren konnten und indem kommunalpolitische Informationen online gestellt wurden (Coleman/Götze 2001, 43f.). Zugleich können solche Informationsangebote auch im verwaltungsinternen Netz oder für Ratsinformationssysteme zur Verfügung gestellt werden (z. B. Stuttgart, vgl. Schwabe/Krcmar 1999). Computeranimationen können aber auch zur Visualisierung in Bürgerversammlungen eingesetzt werden. Im finnischen Tampere konnten die Bürger in Form eines Online-Spieles Planungsalternativen und deren Folgen für die Umgebung und die Landschaft antizipieren und ihre Meinung hierzu abgeben.

Auf der zweiten Ebene der Konsultation werden Bürgerinnen und Bürger zumindest nach ihrer Meinung befragt, die dann auch angemessen berücksichtigt werden muss (nach Abwägung anderer Belange). Neben dem klassischen Beschwerdemanagement zählen hierzu Meinungs- und Kundenumfragen des Stadtmarketings, Anregungen im Rahmen von Bauleitplanverfahren, Wortbeiträge von Bürgern in Gremien, aber auch informelle Verfahren wie Zukunftswerkstätten, Planungszellen oder die Anwaltsplanung. In diese zweite Rubrik fällt das unten vorgestellte Beispiel des OnlineForums in Bremen. Hier weist das Internet vor allem wegen seiner Asynchronität Vorteile auf, d. h. es ist (abgesehen von Chats) nicht notwendig, zu einer bestimmten Uhrzeit an einem bestimmten Ort zu sein.

Als ernstzunehmende Partner werden Bürger (oder die Vertreter von Interessengruppen) in kooperativen Verfahren aktiver Beteiligung begriffen - hierzu zählen viele seit den 70er Jahren und verstärkt durch die Agenda 21 praktizierte informelle Verhandlungsprozesse wie Moderation, Runde Tische und Mediation (vgl. Bischoff/Selle/Sinning 1996). Hier können IuK v. a. durch Gruppenarbeitssysteme (Computer Supported Cooperative Work - CSCW) sowohl den Austausch (und die Archivierung) von Dokumenten erleichtern als auch den Austausch von Argumenten dokumentieren. Die in der Regel förderliche soziale Dynamik von leibhaftigen Gruppenprozessen sollte dann unbedingt mit den Vorteilen der IuK-Anwendungen abgewogen werden. Es wird aber auch deutlich, dass es nicht um ein Entweder-Oder von Offline- bzw. Online-Verfahren geht, sondern oftmals um gegenseitige Ergänzung (,Multi-channel-Strategie“). Wahlen werden an dieser Stelle auch aufgeführt, weil die Bürgerinnen und Bürger bei dieser Beteiligungsform den formal größten Einfluss haben.

Ordnet man nun diese Beteiligungsformen hinsichtlich ihrer politischen Verbindlichkeit und ihres deliberativen Charakters, so kommt man zu einem anderen Ergebnis (vgl. Abb. 1): Die ersten drei Stufen folgen einem Prinzip der Steigerung sowohl bezüglich ihres deliberativen Charakters als auch ihrer Verbindlichkeit, d. h. Information (Kreis) an sich hat keine politische Verbindlichkeit, ist aber Voraussetzung für die beiden nächsten Stufen. Konsultationen (Elipse) können bereits ein recht hohes Maß an Deliberation beinhalten, sind aber noch vergleichsweise unverbindlich, wohingegen aktive Beteiligung (Rechteck im rechten oberen Quadranten) beides einschließt: Ein hohes $\mathrm{Maß}$ an Beratschlagung und Verbindlichkeit. Wahlen hingegen genießen zwar eine hohe politische Verbindlichkeit, sie folgen allerdings keinem deliberativen Modell, weil es nicht um die konstruktive, gemeinsame Lösung bestimmter politischer Probleme geht, sondern eher um Entscheidungen, deren Vorbereitung mehr von Marketinggesichtspunkten als von gemeinsamem Beratschlagen geprägt ist. Bürgerentscheide folgen da zumeist noch eher einem deliberativen Prozess, weil ihnen eine Auseinandersetzung über das zu entscheidende Thema vorangeht. 


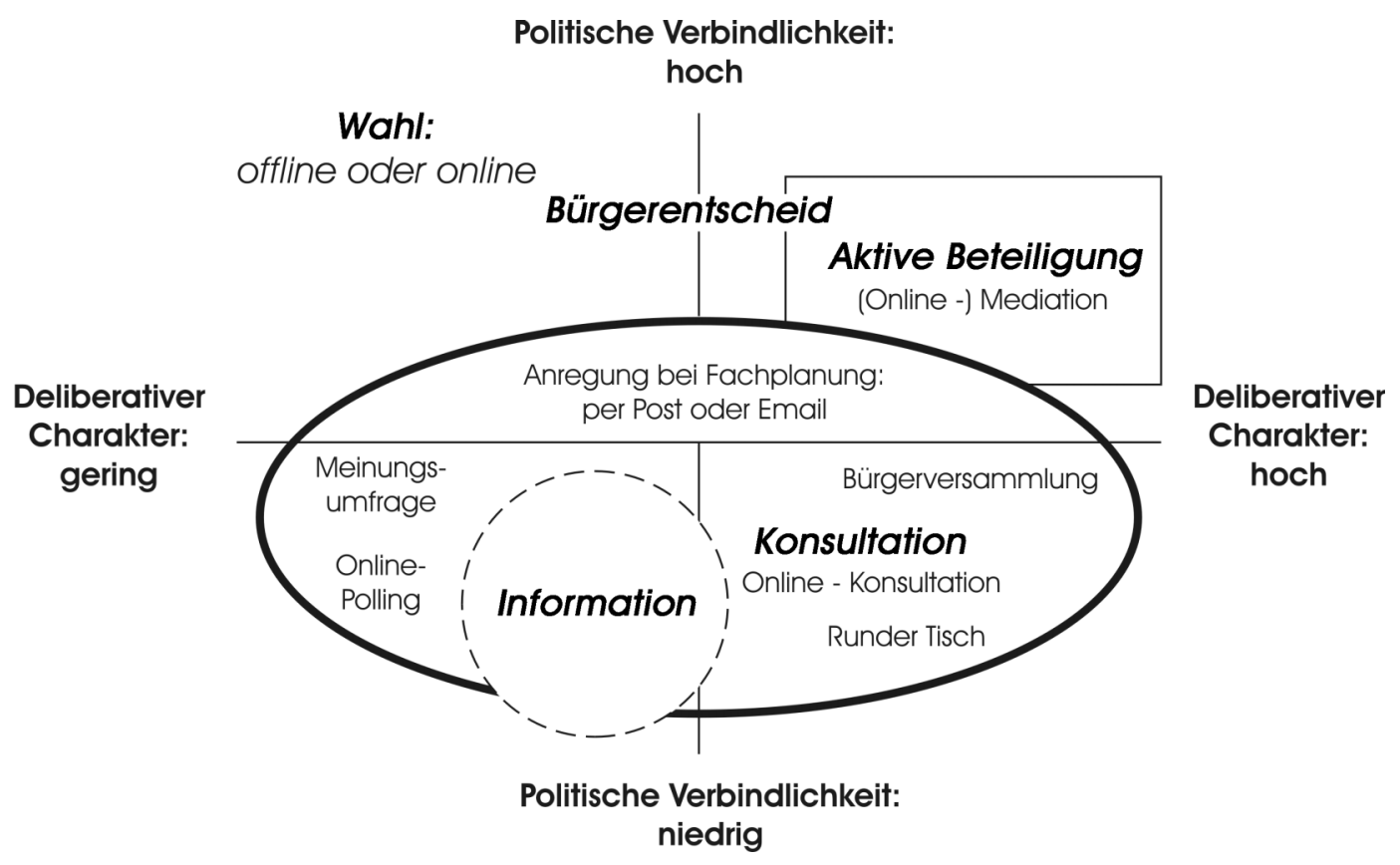

Abbildung 1: Beteiligungsformen hinsichtlich ihrer politischen Verbindlichkeit und ihres deliberativen Charakters

\section{Qualität und Formen der Kommunikation am Beispiel einer Online-Konsultation²}

\section{I Kennzeichen und Nutzungsmöglichkeiten der Online-Konsultation „Forum Horn-Lehe“}

Online-Konsultationen könnten prinzipiell gute Voraussetzungen für deliberative Diskurse ermöglichen - vorausgesetzt sie sind in ein demokratisches Verfahren eingebettet und möglichst vielen bzw. einer repräsentativ ausgewählten Gruppe von Diskutanten offen. Im Rahmen des EU-Projektes E.D.E.N. ${ }^{3}$ bestand die Möglichkeit, in einem Pilot-Projekt im Bremer Ortsteil Horn-Lehe ein

2 Vgl. hierzu die ausführliche Darstellung in Westholm 2002b.

3 Electronic Democracy European Network: Mit dem E.D.E.N.-Projekt soll die Kommunikation zwischen öffentlichen Verwaltungen und den Bürgern in Entscheidungsprozessen verbessert werden. Dieses ambitionierte Ziel soll erreicht werden, indem Tools entwickelt und in vorhandene Infrastrukturen integriert werden, die es den Bürgern ermöglichen, in ihrer Alltagssprache mit der Verwaltung zu kommunizieren („Natural Language Processing). Die innerhalb des E.D.E.N. Projektes in der Entwicklung befindlichen Applikationen beziehen sich auf kommunale (Bauleit-) Planungsprozesse, wobei unterschiedliche Interessengruppen wie Stadtplaner, Architekten, betroffene Bürger einbezogen werden. Die Tools werden dann in den Städten Antwerpen, Bologna, Bremen und Wien in Probeläufen getestet (www.edentool.org). 
Online-Diskussionsforum als eine der zu entwickelnden Internet-Anwendungen zu testen. ${ }^{4}$ Der Versuch hatte folgende wichtige Charakteristika:

- Kombination von Informationen und Diskussion: Um eine qualifizierte Diskussion zu initiieren, wurden entsprechend den von den Nutzern aufgeworfenen Themen Hintergrundinformationen ins Netz gestellt wie z. B. der Flächennutzungsplan (entspricht dem Flächenwidmungsplan in Österreich), Bebauungspläne, Sitzungsprotokolle und eine dreidimensionale Ansicht eines geplanten Baugebietes.

- Breiter inhaltlicher Ansatz - von der Stadt(teil)planung zur Jugendkultur: Um möglichst viele Bürgerinnen und Bürger anzusprechen und für das neue Medium zu interessieren, wurde die Themenpalette breit angelegt. Die beginnende Diskussion im Politiker-Beirat um vielfältige Aspekte der Ortsteilentwicklung wurde als Aufhänger jedes der zur Diskussion stehenden Themenbereiche genutzt.

- Verschiedene Zugangsmöglichkeiten: Die Teilnehmenden am Diskussionsforum sollten sich zunächst registrieren lassen (können), um dann mit richtigem Namen oder mit Phantasienamen (,Nickname“) versehene Beiträge ins Netz zu stellen. Um die Zugangsschwelle möglichst niedrig zu halten, wurde außerdem noch die anonyme Teilnahme ermöglicht.

- Innovation „Bürgermoderation“: Ein erhebliches Problem von Online-Konsultationen bereitet die Moderation. Beiträge und Kommentare müssen thematisch richtig zugeordnet werden, verbale Ausschreitungen, (kommerzielle) Werbung und strafrechtlich relevante Äußerungen müssen unterbunden werden. Umfangreiche Diskussionen müssen nach einiger Zeit zusammengefasst werden. Diese Arbeit ist zeit- und kostenintensiv und kann mit Issue Based Information Systems (IBIS) nur teilweise automatisiert werden. Zudem müssen die User überzeugt sein, dass die moderierende Person neutral und seriös ist, beides kann bei der Verwaltung oder privaten Internet-Firmen unter Umständen angezweifelt werden. Einen Ausweg aus diesen Dilemmata sollte die hier erstmals praktizierte „Bürgermoderation“ aufzeigen, indem im Stadtteil Bürgerinnen und Bürger gewonnen wurden, die nach einer Anleitung diese Aufgabe im Team übernahmen.

Alle interessierten Bürger konnten von der Homepage aus (Abb. 2) „neue Themen vorschlagen“, d. h. innerhalb der vom politischen Beirat vorgegebenen sieben Themenbereiche ${ }^{5}$ Themen aufwerfen, vorhandene „kommentieren“ oder sich per Email direkt an das Moderatorenteam (und die Ortsamtsverwaltung) wenden. Die Kommentare erschienen dabei unmittelbar auf dem Bildschirm, während die neuen „Themen“ erst vom Moderatorenteam überarbeitet werden konnten und zeitlich versetzt online gestellt wurden. Überdies konnten die Nutzer über die Funktion „Dokumente“ Hintergrundinformationen abrufen.

4 Der Ortsteil bot sich auch deswegen besonders an, weil wenige Monate vorher eine Online-Meinungsumfrage ergeben hatte, dass die Bürgerinnen und Bürger starkes Interesse zu haben scheinen, ihre Meinungen und Kenntnisse über dieses Medium anderen mitzuteilen, insbesondere auch gewählten Politikerinnen und Politikern (vgl. Westholm 2002b)

5 Im Laufe der Diskussion wurde von den Organisatoren ein achter Themenbereich „Diskussionsforum“ eingerichtet, in dem v. a. die Technik und Bedienungsfreundlichkeit des Forums diskutiert wurden. 


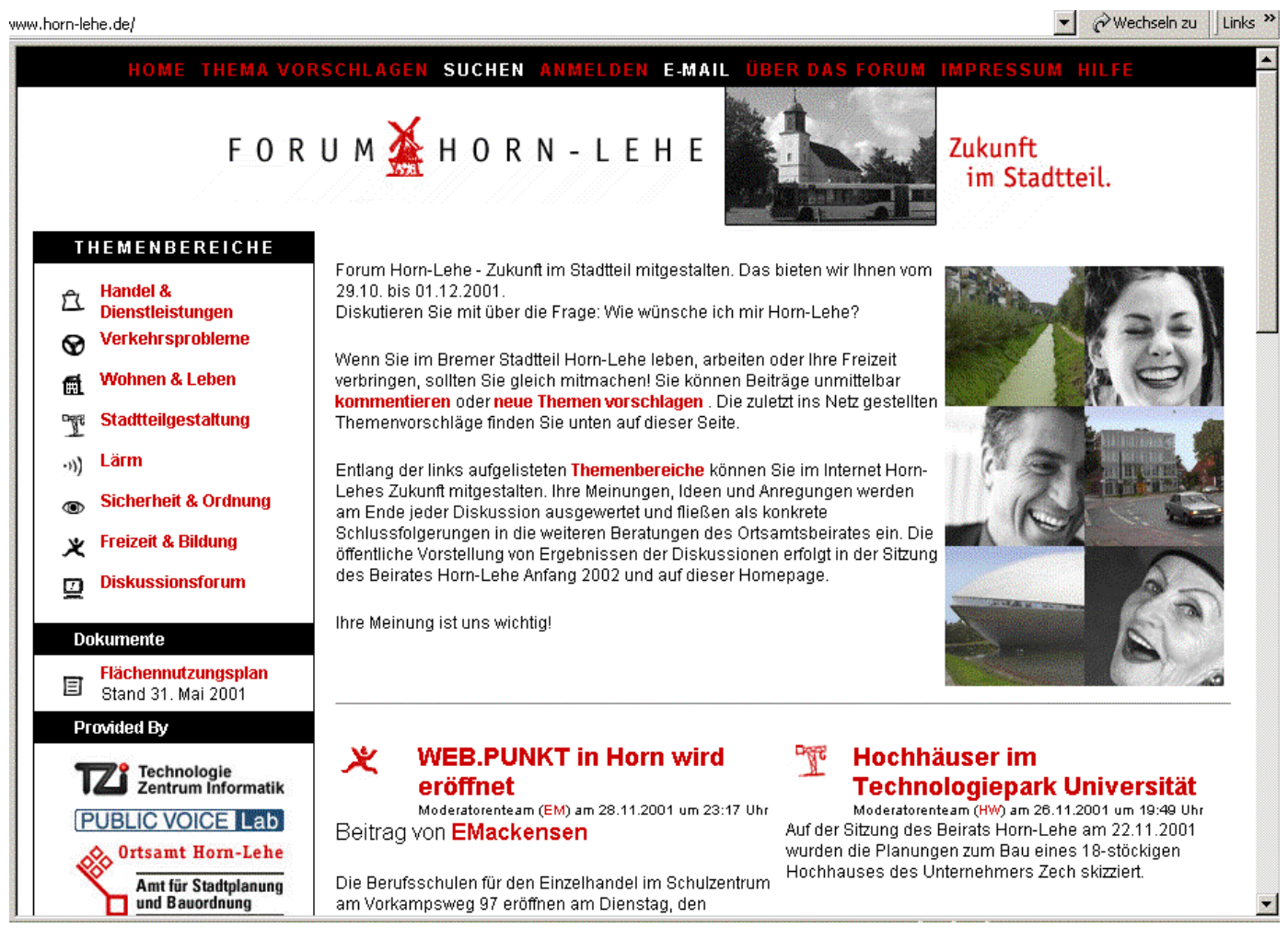

Abbildung 2: Homepage von www.horn-lehe.de nach vier Wochen Laufzeit

Wurde nun z. B. der Themenbereich „Stadtteilgestaltung“ angeklickt, erschien eine Übersicht mit einem Einführungstext, Hintergrunddokumenten sowie einer „Themen“-Übersicht mit Datum und Anzahl der „Kommentare“. Auf diese Weise hatten die User die Möglichkeit, sich zu interessierenden „Themenbereichen“ schnell einen Überblick über die diskutierten „Themen“ zu verschaffen.

Damit ist die zweite Gruppe der „Anwender“ angesprochen, die neben den „Endnutzern“, also den Bürgerinnen und Bürgern, bei Internetauftritten eine wesentliche Rolle spielen. Die Moderatoren gehören normalerweise bei Stadtplanungsforen der Kommunalverwaltung an oder werden von dieser beauftragt. In Horn-Lehe moderierten Bürgerinnen und Bürger ehrenamtlich (unterstützt von der wissenschaftlichen Begleitung), über ihre Technik und Moderationsaufgaben wurden sie in einem halbtägigen Workshop informiert. Aufgaben der Internet-Moderation sind im wesentlichen,

- neu eingegangene Themen zu bearbeiten (einen Aufmachertext zu formulieren; über die Annahme eines neuen Themas zu entscheiden; Werbung und Beiträge mit strafrechtlicher Relevanz zu entfernen $\left.{ }^{6}\right)$;

- „Kommentare“ ggf. mit einer Überschrift zu versehen;

- zu ermuntern (per Email Bekannte oder Interessengruppen-/Fraktionsvertreter etc. anzuschreiben, zu bestimmten Themen Stellung zu nehmen; Nutzer des Forums, von denen aufgrund ihrer bisherigen Beiträge bekannt ist, dass sie zu einem Thema eine andere Meinung vertreten, auffordern, diese nun zum neuen Thema zu äußern);

6 In zwei Fällen sollte Neonazi-Propaganda über die Website lanciert werden, die nichts mit dem Stadtteil zu tun hatte. Diese Beiträge wurden nicht online gestellt. Ein Kommentar, der offensichtlich zur Gewalt (diesmal gegen Neo-Nazis) aufrief, wurde ebenso aus dem Netz genommen. 
- Hintergrundinformationen in elektronischer Form zu recherchieren und online zu stellen;

- Anfragen ggf. an die zuständige Behörde weiterzuleiten, um eine schnelle kompetente Antwort zu bekommen und gleichzeitig Experten aus der Verwaltung einzubinden;

- zu provozieren (Fragen aufwerfen und selber Beiträge schreiben, um die Diskussion zu erweitern);

- zu sortieren (wenn Beiträge verschiedene Themen abdecken, dann diese trennen und verschiedenen Themen zuordnen);

- längere Diskussionen zu einzelnen Themen nach ein bis zwei Wochen zusammenzufassen sowie

- zu schlichten. ${ }^{7}$

Mit der „Bürgermoderation” sollte einerseits Bürgermitwirkung schon auf der Organisationsseite des Forums ermöglicht werden, andererseits war beabsichtigt zu untersuchen, ob so unweigerlich anfallende Kosten für Moderation reduziert werden könnten. Über Zeitungsartikel wurden zunächst Bürger des Stadtteils eingeladen, aufgrund der geringen Resonanz dann einzelne Personen gezielt angesprochen, so dass das Forum letztendlich je zwei Bürgerinnen und Bürger im Alter zwischen etwa 40 und 65 mit Unterstützung des Projektleiters moderierten. Ein wichtiger Grund für alle Moderatorinnen und Moderatoren war (neben dem Interesse am Thema), dass es um ein zeitlich überschaubares Engagement ging. Dieses Experiment glückte, wobei folgende Faktoren für weitere Foren berücksichtigt werden sollten:

- Die „Bürger-Moderatoren” mussten vorher intensiv geschult werden, und zwar sowohl „technisch” als auch „sozio-technisch”, um alle beschriebenen Aufgaben erfüllen zu können. Zum Verständnis der technischen Möglichkeiten ist ein gutes Benutzerhandbuch unabdingbar.

- Gute Erfahrungen wurden damit gemacht, die Moderationsaufgaben nicht tageweise, sondern in 2-4-Stundenblöcken zu vergeben. Auf diese Weise konnten alle die Arbeiten gut in ihren (Arbeits-) Alltag integrieren (allerdings erledigte ein Rentner die meiste Arbeit). Unabhängig davon ist es notwendig, sich über Email und in regelmäßigen (etwa wöchentlichen) Treffen auszutauschen und eine Person im Hintergrund zu haben, die einspringen kann, wenn Probleme auftauchen. Mit unterschiedlichen Erfahrungshintergründen war es auch möglich, inhaltliche Aufgaben (z. B. Zusammenfassungen von Diskussionen) nach Sachkenntnis zu delegieren.

- Die Rolle der Moderation muss auf der Webseite erklärt werden. Es muss klargestellt werden, welche Diskussionsregeln zu beachten sind und über welche Kompetenzen die Moderatoren verfügen; zudem sollte eine (neutrale) Adresse für Beschwerden zur Verfügung stehen. Die Moderatoren schlugen zwar vor, einen „Chefmoderator" zu bestimmen, dies scheint allerdings bei genügendem Austausch innerhalb der Gruppe nicht notwendig und sinnvoll zu sein.

- Es bleibt unklar, ob „Bürgermoderation” bei sehr kontroversen Themen angewendet werden kann (Problem ihrer Neutralität).

- Die Moderatoren können eine wichtige Feedback-Funktion übernehmen und durch ihre Kenntnis von Personen und Wohnumfeld erheblich zum Gelingen eines Forums beitragen, wenn dessen Organisation flexibel genug ist.

7 Bei Mediationen kommen noch weitere Funktionen hinzu wie z. B. die Interessen der Beteiligten herauszuarbeiten, gemeinsame Ziele zu definieren und Win-Win-Lösungen anzustreben (vgl. Zilleßen 1998). 


\subsection{Beteiligung und Inhalt im Online-Forum Horn-Lehe}

6.100 ,Zugriffe” wurden während des Konsultationszeitraums gezählt, wobei nicht Hits auf die Homepage gerechnet wurden, sondern nur solche auf Diskussionen unter den einzelnen Themenbereichen, um möglichst nur solche User zu erfassen, die an den Inhalten interessiert waren und nicht rein zufällig auf die Seite stießen. Interessanterweise stieg die Beteiligung im Zeitverlauf linear, nicht exponentiell, wie man erwarten dürfte, wenn eine zunehmende Zahl von Usern immer mal wieder auf die Seiten schaut, um neue Beiträge (oder auch Reaktionen auf eigene) zu lesen (vgl. Abb. 3). Da man davon ausgehen kann, dass das Forum im Laufe des Diskussionszeitraums bekannter wurde (Zeitung, Mund-zu-Mund Propaganda), kann dies bedeuten, dass die meisten Besucher nicht kontinuierlich die Diskussionen verfolgten, sondern nur ein-, zweimal die Seiten besuchten, aber das Forum nicht als andauernde Website ansahen, auf der man über einen bestimmten Zeitraum (im Beispiel etwa fünf Wochen) Themen berät. Erst dies wäre im Sinne einer idealen Beratschlagung gewesen, bei der man auch auf neue Argumente und Sichtweisen gespannt ist.

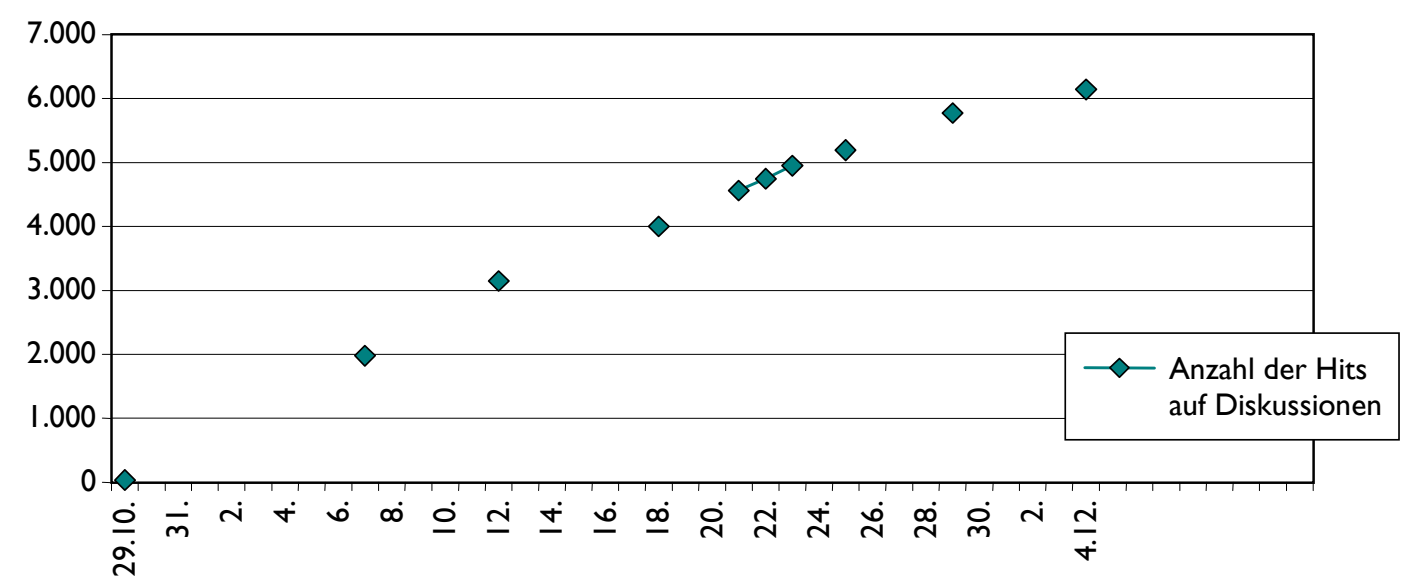

Abbildung 3: (Kumulierte Zahl der) Zugriffe auf die Diskussionen beim Forum Horn-Lehe

Während diese Betrachtung aktiv und passiv Teilnehmende einschließt (vergleichbar den Diskutierenden und Zuhörenden in einer Bürgerversammlung), kommt die eigentliche Diskussion natürlich nur durch aktive Beteiligung zustande. Aus Abbildung 4 ist ersichtlich, dass von den 224 Beiträgen (55 „Themen“ und 169 „Kommentare“) die meisten anonym gepostet wurden. Aus dem Vergleich der Anzahl von mit richtigem Namen und Nickname gekennzeichneten Beiträgen kann man schließen, dass (Teil-)Anonymität zu größerer Lockerheit führt und mehr als nur ein Beitrag geschrieben wird. Die Zahl der anonymen Nutzer kann nur geschätzt werden, überdies sind Überlappungen der drei linken Säulen wahrscheinlich, da sich ein bei der einen Diskussion mit ,richtigem“ Namen registrierter Nutzer durchaus auch an einer anderen anonym oder mit Nickname beteiligen möchte. Insgesamt kann man - in die Sprache von Offline-Beteiligungsverfahren übersetzt - von etwa 1.500 ,zeitweiligen Zuhörern“ und 80 Diskutanten ausgehen. ${ }^{8}$

8 Die Schätzung der 1.500 ,Zuhörer“ („Lurker“) wurde ermittelt durch Subtraktion der Organisationsbeiträge (Technik, Moderation, Evaluation) von der Gesamtzahl (6.100) und anschließende Division durch 3 (bei geschätzten drei Beiträgen pro Person - dieser Wert stellt ein Mittel aus einer großen Anzahl ,zeitweiliger Zuhörer" und wenigen geübten Intensivnutzern dar, die sich kontinuierlich und häufig einloggten. Diese Zahlen - so vorsichtig sie zu genießen sind - sollte man mit der Einwohnerzahl Horn-Lehes (24.000) in Beziehung setzen, um zu weiteren Aussagen über die Repräsentativität von Online-Beteiligungsverfahren im Vergleich zu traditionellen zu gelangen. 


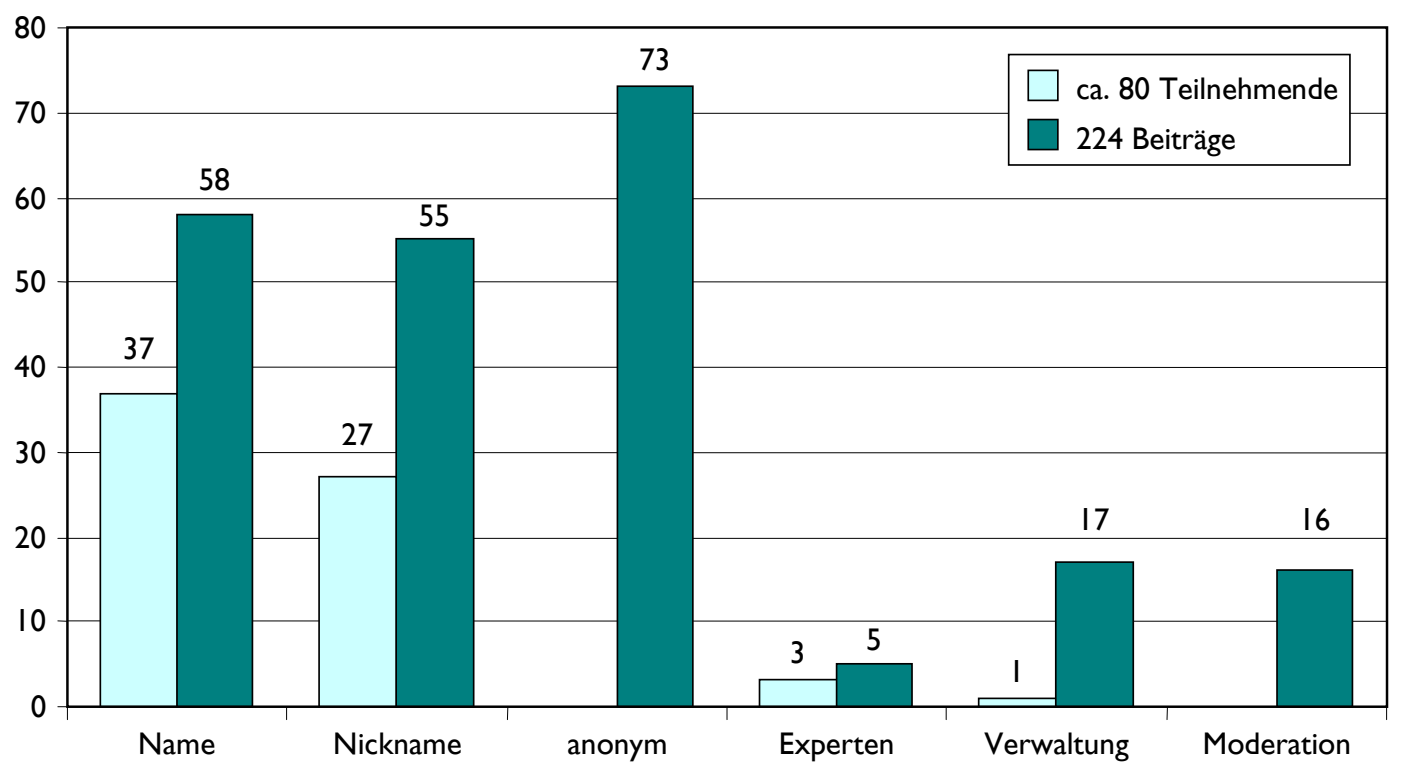

Abbildung 4: Teilnehmende und Beiträge nach Nutzergruppen differenziert

Da das Forum offen sein sollte für die „kleinen Sorgen der Bürger“, überrascht es nicht, dass sich 59 \% der Beiträge auf vor der Haustür liegende Probleme bezogen und (nur) $41 \%$ den Stadtteil als Ganzes betrafen. Vor dem Hintergrund der Klagen von Partizipationsforschern, dass Bürger sich meist nur dann beteiligen, wenn vor ihrer Haustür etwas zu ihrem vermeintlichen Nachteil geschieht (,NIMBY - Not-in-my-backyard! - Problematik), ist der letztere Prozentsatz wiederum vergleichsweise hoch.

\subsection{Qualität und Formen der Kommunikation}

Politische Online-Foren werden u. a. mit dem Anspruch initiiert, noch nicht bekannte Standpunkte in die Diskussion aufzunehmen und vor allem im hier zugrundeliegenden deliberativen Ansatz diese durch Bürgerinnen und Bürger fundiert diskutieren zu lassen. Ein Vorteil von solchen virtuellen Veranstaltungen gegenüber traditionellen könnte sein, dass durch die Asynchronität des neuen Mediums einerseits und die besseren Zugangsmöglichkeiten aufgrund der nicht bestehenden Zeitbegrenzung erst Kommunikation unter mehreren Beteiligten über ein bestimmtes Thema aufkommt. Es stellten sich dann bei der inhaltlichen Auswertung der Forumsbeiträge folgende Fragen: $\mathrm{Ob}$ in den Foren Argumente abgewogen werden; welchen Charakter die Beiträge der User haben, ob sie z. B. nur ihre eigene Meinung ausdrücken wollen oder aber versuchen, die Argumente der anderen zu verstehen, darauf eingehen und sich vielleicht sogar überzeugen lassen.

Im Online-Forum Horn-Lehe gab es (multilaterale) Diskussionen mit vier und mehr (bis zu 17) Kommentaren um 15 Themen (von 55). Eine Voraussetzung, als „Diskussion“ gewertet zu werden, war, dass sich die Postings aufeinander bezogen und sich an einem einzigen Thema orientierten. Um weiterhin zu Aussagen über die Qualität dieser Kommunikation zu gelangen, wurden die Beiträge fünf Kategorien der Kommunikationsforschung zugeordnet (teilweise Mehrfachzuordnungen) (vgl. Leggewie/Bieber 2001):

1. Der Charakter der Beiträge war hauptsächlich expressiv (User drückten ihre Meinung in 210 Fällen aus) (,Expressivität); 
2. 72 Postings bezogen sich direkt auf eine in einem vorangegangenen Beitrag vertretene Meinung (,Reziprozität“);

3. 63 antworteten unmittelbar auf vorangegangene Statements (,Responsivität“);

4. in 10 Beiträgen versuchten die User, sich in die Position des anderen Users hineinzudenken bzw. einzufühlen („Empathie“);

5. Es konnte nicht beobachtet werden, ob User andere überzeugt haben oder sich von deren Argumenten überzeugen ließen („Persuasion”). Allerdings ist dies (nicht nur im Internet) sehr schwer zu erheben; welchen Einfluss Beiträge auf ,passive“ Nutzer haben, die sich nicht mit eigenen Beiträgen beteiligen, entzieht sich jeglicher Beobachtung.

Die Inhaltsanalyse bezüglich des kommunikativen Charakters bestätigt zwar einerseits, dass Foren gerne ausschließlich dazu genutzt werden, der Welt die eigene Meinung mitzuteilen. Andererseits gibt es aber erfreuliche Anzeichen, dass auch „Mehrweg-Kommunikation“ ermöglicht wird, denn die Hälfte aller Kommentare (82 von 169) wurde innerhalb dieser „Diskussionen“ abgegeben. Nun sagt die auf diese Weise kategorisierte Kommunikation untereinander lediglich etwas über die Form, nicht über den Inhalt der Debatten aus.

Der Inhalt und damit die Qualität der Beiträge wurden durch Inhaltsanalyse aller Beiträge beurteilt, indem sie fünf Kategorien zugeordnet wurden:

0 Meinung, Wunsch;

+ Meinung, Vorschlag, Information über einen Mangel;

++ Meinung und konstruktiver Vorschlag, oder Antwort eines Sachverständigen;

- Meinung und unproduktiver Vorschlag;

-- „Meckerkasten”, Beleidigung, Beschimpfung. ${ }^{9}$

Überraschenderweise entpuppten sich anhand dieser Kategorien zwei Drittel der Beiträge als konstruktiv $\left(,+^{\prime \prime}\right.$ und „,++"). Dabei fielen insbesondere mit (richtigem) Namen gezeichnete Beiträge in diese Kategorien, bei den anonymen war es aber immerhin noch knapp die Hälfte. Die Diskussionen profitierten inhaltlich insbesondere von Beiträgen des Ortsamtsleiters und anderer Sachverständiger (vgl. Abb. 5).

9 Ein Beispiel für „0“: „Das gleiche Problem stellt sich in der Millstätter Str.! Durch Anlegung schmaler Gassen in modernen Wohnanlagen löse ich nicht das Problem. Das Auto kann nicht ignoriert werden."

Für „,+“: „Nach meiner Meinung fehlen Fahrradständer an der Haltestelle ,Horner Kirche‘. Platz für diese wäre bspw. zwischen dem Lestra-Parkplatz und dem Fußweg an der Horner Heerstraße."

Für „,+“": „Viele haben es schon erlebt: erst steht man ungläubig, dann immer verärgerter hinter einem Fahrzeug an der Linksabbieger-Ampel der Lestra-Kreuzung [...], vor einem wartet [...] jemand auf grünes Licht der Ampel, das nie kommen wird, wenn, ja wenn man nicht noch bis zur Haltelinie vorfährt. [...] Daher meine Bitte bzw. mein Vorschlag: kann nicht an der Ampel ein deutlich sichtbares Schild angebracht werden: ,Achtung Induktionsschleife, bitte bis zur Haltelinie vorfahren!' Oder die Schleife wird etwas weiter nach hinten verlegt. [...]. Wer spricht mit dem Amt für Straßen und Verkehr? [...]“,

für ,„““ der Kommentar auf das Statement „Haben sich die Verkehrsplaner eigentlich Gedanken darüber gemacht, zu welchen Situationen diese Regelung im Winter führen kann,.. “: , [...] Der Winter wird es an den Tag bringen und die Autowerkstätten werden sich freuen; die Versicherungen weniger!!! Aber vielleicht übernimmt ja der Staat hier die Reparaturkosten. “,

und für „,- -“ der Kommentar auf das folgende Statement: „Der Ausschuss fordert [...] eindeutige Halteverbotszonen auszuweisen. “: „Klar, dann kann man 2-3 mal am Tag einen Streifenwagen durchschicken und Tickets verteilen lassen - typisch Bremen. “ 


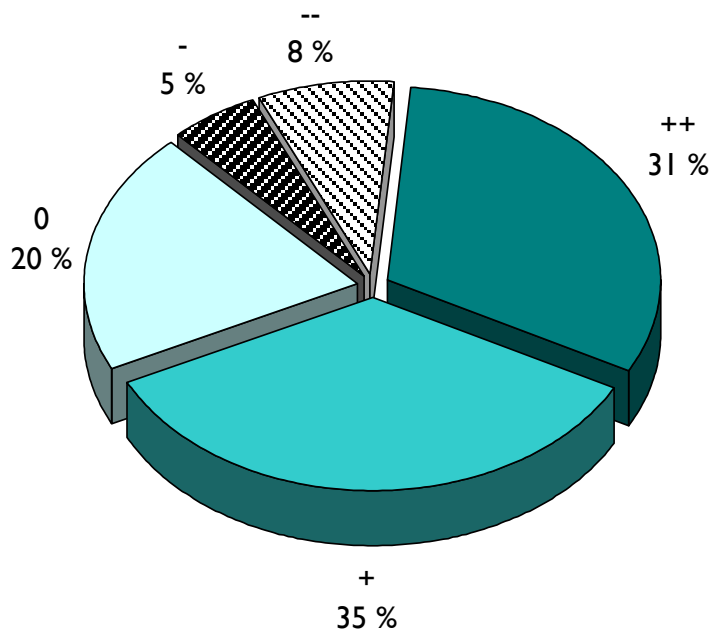

\author{
Abbildung 5: \\ Qualität der Beiträge der \\ Online-Konsultation
}

Bei der Beurteilung der Qualität von Beiträgen werden Politiker und Bürger des Stadtteiles eine andere Meinung vertreten als die Organisatoren des Forums, weil diese aus der Distanz urteilen sie können z. B. vorurteilsfrei an Beiträge „,bekannter Bürger“ herangehen und unterliegen nicht parteipolitischen Voreingenommenheiten, wissen andererseits aber auch nicht, ob es sich bei einem Kommentar um ein lange bekanntes Argument handelt, das die Diskussion nicht voranbringt. Um dieses zu berücksichtigen, wurde den Politikern und Mitarbeitern der Verwaltung auch die Frage vorgelegt, wie sie das Niveau der Beiträge in der Konsultation beurteilten. Hier ergab sich folgende Einschätzung:

- $40 \%$ antworteten gar nicht auf diese Frage;

- Niemand beurteilte die Qualität mit ,verhältnismäßig hoch“;

- $29 \%$ kreuzten an, dass ,überwiegend konstruktive Beiträge” darunter seien;

- weitere $29 \%$ urteilten, dass ,(wenige) konstruktive Beiträge darunter” seien;

- $24 \%$ meinten, das das Forum als „Meckerkasten“ missbraucht würde; ${ }^{10}$

- $10 \%$ hielten das Niveau für ,unterhalb der Beiträge, die in traditionellen Veranstaltungen (z. B. Bürgerversammlungen) gebracht” würden;

- $5 \%$ kreuzten an, dass das Niveau ,sehr niedrig” sei.

(Es konnten mehrere Antworten angeklickt werden.)

Die beiden Beurteilungen der Qualität können nicht im Maßstab 1:1 verglichen werden, da es sich im Falle der wissenschaftlichen Begleitforschung um eine Inhaltsanalyse aller einzelnen Beiträge und nicht um einen verständlicherweise pauschalen Gesamteindruck der User aus Politik und Verwaltung handelt. Es bleiben also Interpretationsunterschiede, die nicht allein mit den oben vorgebrachten Argumenten erklärt werden können. Unterschiede werden auch bleiben, wenn man Wissenschaftler mit diesen Nutzergruppen gemeinsam die Beiträge einschätzen ließe, weil gerade bei der ganz und gar nicht homogenen Gruppe der Politiker der parteipolitische Hintergrund ein wichtiges Moment der Beurteilung darstellt.

10 Wobei der Funktion des Meckerkastens von einem Verwaltungsbediensteten auch Positives abgewonnen wurde: „Es besteht durchaus auch die Chance, dass durch die Funktion des „,Meckerkastens", die das Forum auch, aber ganz sicher nicht nur hat, ein gewisser Aggressionsstau gegenüber der Verwaltung gelöst wird. Vielfach reicht es Betroffenen aus, einfach einmal ihre Meinung sagen zu dürfen, ohne dass sie erwarten, dass ihre Äßßerung sofort und oder immer zu konkreten Handlungen führt.... “. 
Ein wichtiges Ergebnis ist weiterhin, dass eine nicht unerhebliche Zahl von Politikern und Verwaltungsmitarbeitern (40\%) die Beiträge gar nicht gelesen hat. Dieser Befund kann unterschiedlich ausgelegt werden:

- Es ist für solche Untersuchungen gar keine geringe Zahl, heißt es doch im Umkehrschluss, dass die Mehrheit sie (zumindest teilweise) gelesen hat. Zudem gilt bei ehrenamtlich arbeitenden Politikern auch für andere Dokumente, dass sie meist nur von den Key-persons gelesen werden. Zudem gehörten zu der befragten Gruppe auch Landespolitiker, die mit dem Stadtteil keinerlei Berührung hatten.

- Er ist aufgrund der hohen Zahl an Beiträgen erklärlich (etwa 120 Seiten 10-Punkt Text), muss aber Konsequenzen haben für das Content-Management und die Organisation, Aufarbeitung und Zusammenfassung von Beiträgen, denn für die Akzeptanz von Online-Foren in Politik und Verwaltung ist es entscheidend, dass diese Gruppen positive Erfahrungen damit machen, also z. B. für sie relevante neue Erkenntnisse gewinnen und sich der aufzubringende Zeitaufwand lohnt.

\subsection{Erfahrungen mit Online-Beteiligung am Beispiel der Bauleitplanung}

Der unter quantitativen Gesichtspunkten relevanteste und unter dem Gesichtspunkt der Verfahrensintegration komplexeste Beteiligungstyp ist die rechtlich vorgeschriebene Beteiligung an Fachplanungen, die insbesondere

- der Information der Verwaltung einerseits und der Bürger andererseits;

- der Interessenvertretung und dem Interessenausgleich;

- der Effektivitätssteigerung der Verwaltung;

- der Schaffung von Akzeptanz für Entscheidungen sowie

- als vorgelagerter Rechtsschutz und zur Gewährung rechtlichen Gehörs dient (vgl. Fisahn 1996).

Diese prozedurale Form der Beteiligung hat vor allem legitimatorische Funktion für das politische System (vgl. Luhmann 1975). Die wichtigsten und am weitesten entwickelten Formen finden sich in Deutschland, wo die Kommunen einen im europäischen Vergleich besonders starken Stand verglichen mit den anderen Verwaltungsebenen im Staat haben, in der Bauleitplanung in Gestalt der „frühzeitigen Bürgerbeteiligung“ nach Baugesetzbuch und in dem Planfeststellungsverfahren nach Verwaltungsverfahrensgesetz. In den 70er Jahren wurden die (bundesrechtlich fixierten) Möglichkeiten der Bürgerbeteiligung in den Planungsverfahren wesentlich ausgebaut, so dass hier auch die meisten Partizipationserfahrungen vorliegen. Auch wenn es sich hierbei in aller Regel um ,Jedermannbeteiligungen” handelt, stehen die Stellungnahmen der „Träger öffentlicher Belange” (TÖB) im Mittelpunkt der Beteiligung an Fachplanungen, da sich die einzelnen Bürgerinnen und Bürger in der Regel erst mit zunehmender Verfahrensdauer für das Projekt interessieren, gleichzeitig aber die Möglichkeiten zur Einflussnahme mit fortschreitender Verfahrensdauer abnehmen (,Beteiligungsparadox”). „Das Interesse der Bürger an kommunalen Planungen ist leider nicht besonders ausgeprägt, inzwischen finden viele Bauleitplanverfahren quasi unter Ausschluss der Öffentlichkeit statt, oder es werden überwiegend ,Bedenken' statt ,Anregungen' geäußert“ (Schneider 2001). Gleichzeitig werden Beteiligungsmöglichkeiten in jüngster Zeit verstärkt auch in nicht baubezogenen Politikfeldern eingesetzt, z. B. in der Wirtschaftsförderung oder der Kriminalprävention (Frowerk u. a. 1999, Holtkamp 2000) und zum Teil auch gesetzlich vorgeschrieben (z. B. im Jugendhilfebereich in den Kommunalverfassungen einzelner Bundesländer in Deutschland).

Der Bereich der Bauleitplanung ist dabei aber derjenige, bei dem am stärksten im eigenen Wirkungskreis die Entwicklung der Kommune beeinflusst werden kann. Erste Schritte der IuK-Unter- 
stützung werden seit einiger Zeit mit der Bereitstellung von Bebauungsplänen und Flächennutzungs- bzw. Flächenwidmungsplänen im Internet unternommen. So stellen z. B. Wien und Leipzig inzwischen ihre Bebauungspläne vom Aufstellungs- bis zum Satzungsbeschluss z. T. dreidimensional (Darmstadt) für ihre Bürger im Netz zur Verfügung. In Bonn und dem umgebenden RheinSieg-Kreis können sich Bau- und Planungsinteressierte Stadtpläne, topographische Karten und Luftbilder anschauen und in übersichtlicher Form Infrastruktureinrichtungen wie z. B. Schulen, Kindergärten, Bahnverbindungen, Straßen sortiert nach „Basisinformationen“ und „planungsrelevanten Informationen“ nach Bedarf darauf anzeigen lassen. ${ }^{11}$ Düsseldorf $\mathrm{z}$. B. verfügt inzwischen über mehrjährige Erfahrung mit der Online-Beteiligung von Bürgerinnen und Bürgern in Bauleitplanverfahren. Angeboten werden Informationen zur Planung (mit Karte, Glossar, Erläuterungstext, Grünordnungsplan, fotografischer Ansicht des gegenwärtigen Zustands). Überdies können die Nutzer über eine Https-Verbindung (d. h. mit Sicherheitszertifikat) Anregungen zur Planung abgeben, wobei sich die Stadt selbstverpflichtete, diese „Anregungen ... bei der weiteren Bearbeitung der Planverfahren in die vorzunehmende Abwägung der betroffenen Belange“ einzubeziehen. Neben der Anregung müssen nur Felder ausgefüllt werden, aus denen ersichtlich ist, ob der Nutzer z. B. im Plangebiet wohnt oder allgemein als Planungsinteressierter, Eigentümer oder Mieter auftritt. Die Angabe von Email-Adresse, Name und Anschrift erfolgt freiwillig. ${ }^{12}$

Für die bilaterale Kommunikation mit der Verwaltung eignet sich insbesondere das Forum mit seiner Möglichkeit der zeitversetzten Antwort, wobei hier vielfach erst noch die Zuständigkeit und das genaue Verfahren innerhalb der Verwaltung geklärt werden müssen. Im Internet geschieht hierzu einiges: In Osnabrück z. B. werden bereits seit 2000 Erfahrungen gesammelt, als der Vorentwurf und später der Entwurf des Flächennutzungsplanes ins Internet gestellt worden waren. Die Bürgerinnen und Bürger konnten per Email Stellungnahmen abgeben und dabei darüber entscheiden, ob ihre Kommentare online veröffentlicht werden sollen. Die Karte des Plans war interaktiv nutzbar, indem Informationen zum angeklickten Objekt angezeigt wurden, Kartenausschnitte verkleinert bzw. vergrößert oder Bildausschnitte verschoben werden konnten. Gleichzeitig wurde die Möglichkeit gegeben, sich in einem Forum an der Diskussion zu beteiligen; hier reagierte das Planungsamt auf Stellungnahmen auch unmittelbar im Netz. Eine empirische Begleituntersuchung zur Akzeptanz dieses Angebotes kam zu dem Schluss, dass die Organisations- und Artikulationsfunktion des Internet momentan noch eine untergeordnete Rolle spielt, bisher nicht interessierte Bevölkerungsschichten auch nicht übers Internet gewonnen werden, sondern eher die kommunikationsstarke Elite das Internet zur Information benutzt (vgl.Hohn 2001).

Die Stadt Esslingen hat in ihrem Konzept zum (deutschen) Multimedia-Städtewettbewerb MEDIA@Komm den Schwerpunkt auf die technik-unterstützte Beteiligung der Bürgerinnen und Bürger gelegt. Unter anderem konnte online über das Baugebiet „Zeller Egert“ diskutiert werden. Die Bürgerinnen und Bürger konnten nicht nur Dokumente einsehen (Pläne, Statistiken, Folien, Fotos, Skizzen, Protokolle und Verweise auf externe Informationen, wie z. B. auf die Website einer Gegeninitiative), sondern auch während einer vierwöchigen „Internet-basierten Bürgeranhörung“ ihre Meinung unter eigenem oder Phantasienamen in einem moderierten Diskussionsforum äußern. Die drei (externen) Moderatoren bereiteten die Themen auf, strukturierten die Diskussion, fassten den Diskussionsstand zusammen und sicherten die Ergebnisse. Dabei wurde deutlich, dass eine (unabhängige) Moderation einen zentralen Erfolgsfaktor internet-basierter Bürgerbeteiligung darstellt, damit aber erheblicher Aufwand verbunden ist. Ebenfalls wurde deutlich, dass ohne eine glaubwürdige Anbindung an das formale Verfahren der aufwendige Einsatz einer Online-Informationsund Kommunikationsplattform ins Leere zu laufen droht, so dass der Einbettung in den Verfahrenskontext besondere Aufmerksamkeit zukommen muss (vgl. Trénel u. a. 2001).

11 Vgl. www.umzug-nach-bonn.de.

12 Vgl. https://www.duesseldorf.de/planung/meinung/ssl_anregung.shtml (Download 17.11.2002) 
Bei fachlich komplexeren und mehrere unterschiedliche Interessen tangierenden Planungen und Entscheidungen reichen ein Forum oder eine Umfrage in der Regel nicht aus, um den Bürgerwillen herauszufinden oder einen Konsens bzw. Kompromiss zu erzielen. Verhandlungsverfahren, die die Betroffenen bzw. ihre Vertreter möglichst frühzeitig in Planungen einbeziehen, können nicht nur die Akzeptanz des Ergebnisses erhöhen, sondern auch die im Gemeinwesen schlummernden Kenntnisse ans Tageslicht fördern und die Ergebnisse dadurch optimieren. Diese Verfahren gewannen in den 90er Jahren im Zuge der Kritik an der Unzulänglichkeit traditioneller Planungsverfahren an Bedeutung. In diese Kategorie fallen informelle, zumeist konsensorientierte Verfahren, die von Politik und Verwaltung entweder mit Repräsentanten von Interessengruppen (Mediation, meist auch Runde Tische) oder mit Einzelpersonen projektbezogen (z. B. Foren, Zukunftskonferenzen oder Planungszellen) durchgeführt werden. ${ }^{13}$ Technisch lassen sich Verhandlungsprozesse mit elektronischen Diskussionsforen oder CSCW-Anwendungen abbilden oder unterstützen. Auch für anspruchsvollere Beteiligungsverfahren wie die Mediation existieren inzwischen technische Anwendungen. Ein Beispiel dafür ist das vom Fraunhofer Institut Autonome Intelligente Systeme entwickelte Produkt Zeno (vgl. Trénel u. a. 2001). Aber auch hier gilt, dass die Technik nicht der entscheidende Faktor ist. Die Verfahren erfordern von den (ehrenamtlich) beteiligten Bürgerinnen und Bürgern (auch wenn sie bestimmte Interessengruppen repräsentieren) zumeist ein hohes Maß an Engagement über einen längeren Zeitraum, führen keineswegs immer zu einem Konsens, und selbst dieser ist letztlich rechtlich nicht verbindlich. Allerdings setzen sich die politisch Verantwortlichen in ein schlechtes Licht, wenn sie die Ergebnisse ohne einsichtige Gründe nicht umsetzen, so dass man von einer hohen politisch-moralischen Verbindlichkeit der in solchen informellen Verhandlungsprozessen erzielten Ergebnisse sprechen kann.

13 Vgl. Bischoff/Selle/Sinning (1996); einige hierunter auch zu fassende Verfahrenstypen, wie z. B. die Planungszelle, die Zukunftswerkstatt oder die Anwaltsplanung, wurden bereits in der ersten Beteiligungswelle in den 70er Jahren angewendet. 


\section{Anschlussfähigkeit von eDemocracy}

Die wenigen vorliegenden Erfahrungsberichte zeigen, dass die bloße technische Möglichkeit, eine bestimmte Variante der Bürgerbeteiligung per Internet abzuwickeln, keine Effekte auf die inhaltlich-substantielle Beteiligung hat. Die große Herausforderung liegt darin, solche technischen Konstrukte in den jeweiligen organisatorischen, kulturellen, rechtlichen und ökonomischen Kontext einzubetten und politische Unterstützung dafür zu bekommen. Anders ausgedrückt müssen die elektronischen Formen in der sog. virtuellen Welt anschlussfähig an die Prozesse und Strukturen in der realen Welt gemacht werden - der Seite der Anwender in Politik und Verwaltung und der Seite der Endnutzer, Bürger und Interessenvertreter. Insgesamt muss diese Anschlussfähigkeit im Hinblick auf die im folgenden aufgezeigten Faktoren hergestellt werden.

\section{I Technische Anschlussfähigkeit}

In technischer Hinsicht müssen sich die Online-Beteiligungsangebote in die jeweilige Infrastruktur wie auch in die Fachverfahren integrieren lassen. Noch handelt es sich überwiegend um befristete und isolierte Projekte, in denen zu einem bestimmten Anlass, oft mit externer Unterstützung und zusätzlichen Mitteln, Planungsunterlagen ins Netz gestellt werden. Diese werden häufig nicht direkt aus dem internen Planerstellungsprozess heraus produziert und aktualisiert, so dass bei der nächsten Planerstellung alles wieder von vorne beginnt. Dies gilt für die Bauleitplanung ebenso wie für die Wählerverzeichnisse bei Online-Wahlen. Dabei sind im einzelnen durchaus langwierige Abstimmungen und Anpassungen vorzunehmen.

Gleichzeitig dürfen die technischen Anwendungen nicht die Leistungsfähigkeit der Nutzer bzgl. Hard- und Softwareausstattung überfordern. So verfügen sie häufig nicht über einen entsprechend leistungsstarken Internetzugang (DSL, mindestens aber ISDN) und müssen dann für Anwendungen und Daten aus dem geographischen Bereich lange Downloadzeiten in Kauf nehmen. Erklärungen zum Herunterladen anderer Anwendungen müssen z. B. unbedingt muttersprachlich erfolgen und auf einer Vertrauen einflößenden Webseite. Überdies sollte die Grundregel des WYSIWYG (What you see is what you get) eingehalten werden, wenn die Nutzer aufgefordert sind, selber Themen beizusteuern und zu kommentieren: User möchten auch gerne mal etwas in ihren Beiträgen unterstreichen oder kursiv setzen, das muss möglich sein. Zudem muss ihnen auch der Grad der mehr oder weniger geschützten Übermittlung von Daten erläutert werden.

Oft kann es auch angebracht sein, nicht nur IuK-Technologien einzusetzen, sondern Online-Anwendungen stärker in ihrer Ergänzungsfähigkeit zu Offline-Verfahren zu sehen: Bei der oben erwähnten (Internet-) Umfrage fiel auf, dass sich - gemessen am Internetzugang - viele ältere Menschen (32,5\% gaben an, „60 Jahre und älter“ zu sein) beteiligt haben. Hier zeigt sich, dass es sinnvoll war, bei der anvisierten Zielgruppe den Fragebogen nicht nur online zur Verfügung zu stellen, sondern mehr-kanalig (,multi-channel“) vorzugehen und ihn auch als gedruckte Version im Ortsamt zum Ausfüllen auszulegen, so dass sich auch Menschen zu Wort melden konnten, die nicht das Internet nutzen - z. B. machten besonders die Bewohnerinnen und Bewohner eines Altenheimes von dieser Möglichkeit Gebrauch. Eine solche Internet-Befragung sollte also möglichst nur als Ergänzung zu anderen Beteiligungsformen genutzt werden (Bürgerversammlung, gedruckter Fragebogen etc.), nicht als mittelfristiger Ersatz. Gerade dieses ,multikanalige“ Vorgehen könnte zu größerer und qualifizierter Beteiligung führen. Für Bebauungsplanverfahren ist in Tabelle 2 beispielhaft dargestellt, wie eine solche mehr-kanalige Vorgehensweise aussehen könnte. 
Das Zusammenwirken verschiedener Medien ist aber noch in anderer Weise sinnvoll: Nicht nur die Kombination Internet-Abstimmung mit Papierfragebogen führte im Beispielfall der Meinungsumfrage zu hoher Beteiligung, auch die Zusammenarbeit mit der vielgelesenen örtlichen Zeitung war unbedingt notwendig: Nachdem hier bekannt gemacht wurde, dass eine solche Umfrage stattfindet, gab es einen ersten Beteiligungs"run"; nach der Veröffentlichung des Zwischenergebnisses in der Zeitung folgte der nächste Schub.

Tabelle 2: Multi-channel Bürgerbeteiligung am Beispiel von Bebauungsplanverfahren

\begin{tabular}{|c|c|c|c|}
\hline \multicolumn{2}{|c|}{ Traditionelle „Offline“-Beteiligungsschritte } & \multicolumn{2}{|c|}{ eDemocracy-Tools } \\
\hline Formell & Informell & Als Ergänzung & Als Ersatz \\
\hline $\begin{array}{l}\text { Tagesordnungen der } \\
\text { Bauausschusssitzungen in } \\
\text { der Tagespresse }\end{array}$ & & $\begin{array}{l}\text { Email-Benachrichtigung z. B. } \\
\text { über „Bürgerbrief“" der Kom- } \\
\text { mune an interessierte Bürger }\end{array}$ & $\begin{array}{l}\text { Abhängig von der } \\
\text { Internetpenetration } \\
\text { in der Bevölkerung }\end{array}$ \\
\hline $\begin{array}{l}\text { Zuhören im Bauausschuss } \\
\text { jeweils vor Aufstellungs-, } \\
\text { Auslegungs- und } \\
\text { Satzungsbeschluss }\end{array}$ & $\begin{array}{l}\text { Rederecht für } \\
\text { Nicht-Mitglieder }\end{array}$ & & \\
\hline $\begin{array}{l}\text { Frühzeitige und reguläre } \\
\text { Bürgerbeteiligung: Hinweis } \\
\text { in Tageszeitung }\end{array}$ & & $\begin{array}{l}\text { Email-Benachrichtigung z. B. } \\
\text { über „Bürgerbrief“" der Kom- } \\
\text { mune an interessierte Bürger }\end{array}$ & $\begin{array}{l}\text { Abhängig von der } \\
\text { Internetpenetration } \\
\text { in der Bevölkerung } \\
\text { und Änderung } \\
\text { rechtlicher Bestim- } \\
\text { mungen (zumeist } \\
\text { Landesrecht) }\end{array}$ \\
\hline $\begin{array}{l}\text { Frühzeitige Bürgerbeteiligung: } \\
\text { Auslegung im Amt für } 4 \\
\text { Wochen: (schriftliche) } \\
\text { Anregungen }\end{array}$ & & $\begin{array}{l}\text { Pläne im Netz: Anregungen } \\
\text { per Email }\end{array}$ & \\
\hline $\begin{array}{l}\text { Ggf. Bürgerversammlung } \\
\text { nach Auslegungsbeschluss }\end{array}$ & $\begin{array}{l}\text { Frühzeitige } \\
\text { Bürgerbeteiligung: } \\
\text { Bürgerversammlung }\end{array}$ & $\begin{array}{l}\text { Visualisierung der Planung } \\
\text { mit 3-D-Animationen in der } \\
\text { Versammlung }\end{array}$ & \\
\hline
\end{tabular}

\subsection{Rechtliche Anschlussfähigkeit}

Dies korrespondiert unmittelbar mit der rechtlichen Anschlussfähigkeit: Nutzer müssen über ihre eigenen Schutzrechte (z. B. bzgl. des Datenschutzes) aufgeklärt werden. Auf Seiten des politischadministrativen Systems ist die rechtliche Zulässigkeit aufgrund bestehender oder zu novellierender Vorschriften eine unabdingbare Voraussetzung. Dabei geht es keineswegs nur um das Signaturgesetz, sondern um eine Fülle fach- und verfahrensrechtlicher Vorschriften in fast allen oben angesprochenen Feldern der Beteiligung.

Bei dem der oben ausführlich geschilderten Online-Konsultation vorangegangenen Polling (vgl. Westholm 2002a) z. B. wurden keinerlei sicherheitstechnische Maßnahmen vorgenommen wie z. B. Registrierung der IP-Nummer oder eine andere Form der Nutzerregistrierung - mit dem Vorteil des Datenschutzes durch Anonymität. Da Bremen Vorreiter beim Einsatz der digitalen Signatur für Verwaltungstransaktionen ist, wäre es auch vorstellbar gewesen, diese einzusetzen. Dieses Verfahren wurde hier aber derzeit noch für zu aufwendig, nutzer-unfreundlich und unflexibel gehalten; wegen der dafür erforderlichen Umstände hätte es dementsprechend die Zahl der Beteiligten erheblich ge- 
senkt. Stattdessen wurde in dem betreffenden Beispiel über den Einbau einer offenen Frage (wer würde sich schon die Mühe machen, mehrmals abzustimmen, aber sich jedes Mal einen anderen Text auszudenken?) und den Abgleich der Einzelwertungen auf Plausibilität und inhaltliche Abweichung versucht, Mehrfachabstimmungen und unrichtige Angaben zum Wohnort und zum Alter auf ein Minimum zu reduzieren. Dies ist, wie spätere Interviews und Gespräche ergaben, nur eingeschränkt gelungen: So wurde von einzelnen durchaus mehrfach abgestimmt, und zwar sehr phantasievoll: Im Stundenrhythmus wurde der Fragebogen am Arbeitsplatz aufgerufen, die wichtigste inhaltliche Frage dann immer identisch beantwortet, alles andere, insbesondere der Wohnort, aber abgewandelt.

Ein weiteres Problem für Abstimmungen besteht darin, dass besonders interessierte Kreise (Bürgerinitiativen, andere Interessengruppen, in diesem Beispiel ein Sportverein oder ein Seniorenheim) verständlicherweise ihr Klientel zur Teilnahme motivieren, so dass kaum Repräsentativität gewährleistet ist (ein ähnliches Phänomen wie bei Bürgerbegehren, also unabhängig von der eingesetzten Technik und dem betriebenen Sicherheitsaufwand). Für die Online-Konsultation wurden ähnliche Bedenken geltend gemacht, z. B. von einem Verwaltungsvertreter: „Die starke Fokussierung auf die wenigen Äußerungen, die keineswegs eine Mehrheitsmeinung darstellen, führen leicht zur Verzerrung des Bildes der öffentlichen Meinung. Dies führt - ähnlich wie die Leserbriefe - zu Meinungsverzerrungen, die im administrativen Bereich schwer aufgefangen werden können. Die Tatsache, sich selber - häufig auch anonym - melden zu können und die schriftlichen Eingaben dann für alle lesbar zu machen, führt bei manchen VerfasserInnen zu falschen Einschätzungen über die Relevanz ihrer Eingaben. Es wird erforderlich sein, sich mit diesem Medium erst vertraut zu machen." Überdies wurde von einigen Horn-Leher Politikern auch die eingeschränkte Zugangsmöglichkeit zum Internet als Argument mangelnder Repräsentativität des Forums und damit verminderter Geltungskraft der dort gemachten Aussagen und Vorschläge aufgeführt. Nur wundert es, dass dieses Argument bei traditionellen Beteiligungsverfahren nicht ins Gewicht fällt. Dass den Politikern viele Namen der Diskutanten bekannt waren, wurde als weiteres Argument für geringe Repräsentativität ins Feld geführt.

\subsection{Einbettung in bestehende Organisationszusammenhänge}

Die Einbettung in bestehende Abläufe und Verfahren sowie die Berücksichtigung von Zuständigkeitsregelungen machen einen wesentlichen Bestandteil der organisatorischen Anschlussfähigkeit auf Seiten der Verwaltung aus. Diskurs-basierte Beteiligungsangebote sind wenig glaubwürdig, wenn eine klare Verwertungszusage fehlt. eDemocracy-Angebote müssen - genauso wie andere Formen informeller Beteiligung - in den politischen Prozess eingebunden sein. Auf Nutzerseite gilt es, z. B. bei Assoziationen ggf. interne Abstimmungsverfahren zu berücksichtigen.

Online-Beteiligungsverfahren unterliegen derzeit noch der Gefahr, v. a. der Profilierung bestimmter Institutionen und Personen als der Sache zu dienen, so dass zwischen Anwendungen aus Selbstzweck und sinnvoller Einbettung in politische Prozesse unterschieden werden muss (vgl. Kubicek/ Westholm 2002). Die Bremer Online-Konsultation z. B. wurde an den Anfang des Beratungsprozesses im politischen Gremium gelegt, als noch keine Entscheidungen fielen, sondern nach Vorschlägen und Ideen für neuralgische Punkte im Stadtteil gesucht wurde und Entscheidungsvorbereitungen getroffen wurden. Die Politiker wurden über das Geschehen im Forum auf dem Laufenden gehalten (Politikern ohne Internetanschluss wurden z. B. alle Beiträge auf Papier ausgedruckt zur Verfügung gestellt). Dass parallel noch andere Formen der Bürgerbeteiligung geplant wurden (Runder Tisch und Fragebogenaktion), macht die Aktion noch glaubwürdiger. Dass Online-Aktivitäten auch völlig an politischen und verwaltungsmäßigen Verfahren vorbeilaufen können, zeigte das Esslinger Beispiel, bei dem der Rückfluss in die politischen Gremien gar nicht funktionierte. 
Beim Bremer Forum wurde darüber hinaus ein Kernproblem von Online-Foren offenkundig, das der begrenzten Informationsverarbeitungskapazität von Politik und Verwaltung. Beim Forum wurde von der wissenschaftlichen Begleitung bewusst darauf verzichtet, Zusammenfassungen zu schreiben, um gerade für dieses Problem eine nachhaltige Lösung - auch mithilfe der angesprochenen Politiker und Verwaltungsmitarbeiter - zu finden. Hier litt das Forum an seinem Erfolg, dass sich viele Bürgerinnen und Bürger beteiligten und sehr viel Material zusammentrugen, das von irgendjemandem strukturiert werden muss, um von Politik und Verwaltung aufgenommen werden zu können. Content-Management von Forum-Beiträgen muss in das Blickfeld geraten, wenn Foren mehr sinnvolle Anwendungsfelder finden sollen. Hierzu zählt auch, kurze Wege zu schaffen vom Forum direkt zu den sachbearbeitenden Verwaltungsstellen, die entweder eine Bürgerfrage beantworten oder bei einem Mangel Abhilfe schaffen können.

\section{4 Ökonomische Anschlussfähigkeit}

Entscheidend unter den derzeitigen finanziellen Rahmenbedingungen der öffentlichen Hand und hier insbesondere der Kommunen ist vor allem die Gewährleistung der ökonomischen Anschlussfähigkeit. Das heißt vor allem die Sicherstellung der Finanzierbarkeit der Entwicklung und des regelmäßigen Betriebs. Anwendungen, die nur äußerst selten benötigt werden, aber aufgrund ihrer Komplexität (Wahlfunktionalitäten z. B.) oder der begleitenden Maßnahmen (Werbung, Moderation, Aufbereitung der Anregungen für die Politik bei Online-Foren) sehr kostenintensiv sind, sind in diesem Sinne nur bedingt anschlussfähig. Im erweiterten Sinne schließt es aber auch eine prinzipielle Kosten-Nutzen-Analyse auf Seiten der Endnutzer wie der Verwaltung ein. Dann muss auch die Motivation aller Beteiligten berücksichtigt werden: Z. B. ist die Motivation der Anwender in den Verwaltungen entscheidend für den Erfolg. Zur Demotivierung kann hier die personelle Ausstattung führen und die Befürchtung, durch mehr Beteiligung noch mehr Arbeit aufgebürdet zu bekommen. Insbesondere für Webforen ist dies offenkundig, da hier bei auf multilaterale Kommunikation angelegten Diskussionsplattformen aufwendige Moderationen unabdingbar sind.

Bürgerbeteiligung wird nur dann zustande kommen, wenn die Adressaten motiviert sind, das Angebot zu nutzen. Die Mitwirkungsbereitschaft der Bürgerinnen und Bürger wird in vielen Fällen überschätzt. So zeigen Befragungsergebnisse immer wieder, dass politische Inhalte unter den bevorzugten Internet-Angeboten einen hinteren Platz einnehmen, was allerdings nicht verwundert, wenn man sich das (in den 70er Jahren durch empirische Politikforschung entwickelte) ,Standardmodell der Beteiligung“ vergegenwärtigt: Wer gut situiert ist und eine entsprechende Stellung in der Gesellschaft hat, wird eher glauben, mit eigenem politischen Engagement etwas bewirken zu können als andere (vgl. Dalton 1988). Dies ändert sich nicht, nur weil eine neue Technik eingesetzt wird. Hoffnungen, neue Zielgruppen zu erschließen, haben sich bislang nicht erfüllt. Jugendliche z. B. werden sich allenfalls für solche Fragen der kommunalen Fachplanungen interessieren, die sie selbst betreffen, also z. B. für den Neubau eines Jugendzentrums. Am erfolgreichsten sind die Fälle, in denen existierende Diskussionen oder kontroverse Planungen auch mit Hilfe des Internet diskutiert werden können. Das heißt, die technik-gestützten Angebote müssen den Anschluss an die bestehenden Kontroversen und Motivationen suchen.

Auf Nutzerseite können gerade organisierte Interessen ihre Meinungen in Pollings und Foren gut platzieren: Die eigene Klientel kann hier über Email schnell benachrichtigt und zur Teilnahme mobilisiert werden. Da sich nicht Massen beteiligen, läuft man Gefahr, schnell bestimmte Gewichtungen zu provozieren. Um den Anreiz für einzelne Teilnehmer zu erhöhen, sollte auch darüber nachgedacht werden, in Diskussionsforen Meinungsumfragen zu integrieren, die einzelne Diskussionen im Netz begleiten (und ggf. beenden). 
Auf Anwenderseite müssen die Kosten und Nutzen solcher Verfahren gegeneinander abgewogen werden. Zu den Kosten gehören nicht nur die für die Technik, sondern auch die Arbeitszeit der Verwaltungsmitarbeiter (und Politiker), die die Diskussion moderieren und die Beiträge lesen und ggf. darauf reagieren müssen: „Es entsteht ein Mehraufwand und i.d.R. auch eine Zeitverzögerung, wenn abweichende Meinungen zur Bürgerversammlung oder Politik dominierend sind“, äußerte sich ein Vertreter der Verwaltung, der damit nicht alleine steht, dies zeigten auch im Rahmen des E.D.E.N.Projekts zu den Nutzeranforderungen mit Planern in der Verwaltung geführte Interviews. ${ }^{14}$ Das dem oft gegenübergestellte Argument, dass mehr Offenheit gegenüber Bürgeranliegen mittelfristig bessere Akzeptanz von Planungen schaffe, hilft dem Sachbearbeiter in der Verwaltung wenig, der ein Forum auswerten muss. Die guten Erfahrungen mit der ,sozialen Innovation“ der „Bürgermoderation“ ist ausbaufähig und könnte sowohl Kosten sparende als auch sozial integrierende Funktion haben.

„Motivation“ ist ein entscheidender Punkt, Online-Aktivitäten in der Verwaltung zu forcieren oder zu bremsen. Um die eigenen Mitarbeiter zu motivieren, ist es deshalb sehr sinnvoll, wenn Politik und Verwaltungsspitze hinter solchen Aktivitäten stehen und auch den Arbeitsaufwand zu würdigen wissen - ohne engagierte und motivierende Persönlichkeiten gelingt keine ökonomische Anschlussfähigkeit.

\subsection{Kulturelle Anschlussfähigkeit}

Unter kultureller Anschlussfähigkeit werden hier vorhandene Qualifikationen und Rollenverständnisse (z. B. Hoheitsverwaltung vs. Dienstleistungsorientierung, passiver vs. aktiver Staatsbürger) wie auch vorherrschende Leitbilder auf Anwender- und Nutzerseite gefasst, ob es z. B. eine „Beteiligungskultur“" in der betreffenden Kommune gibt. Auch die Einstellungen zur Technik, gesellschaftliche Experimentierfreude und Sicherheitsängste fallen unter diese Rubrik.

Um glaubwürdig zu sein und Bürgerengagement nicht leichtfertig für die Zukunft aufs Spiel zu setzen, sollten eDemocracy-Angebote in eine entsprechende Kultur der Beteiligung eingebettet sein. Bürgerinnen und Bürger sollten wissen, dass ihre Meinung auch schon in anderen formellen und informellen Verfahren von den Gemeinderäten ernst genommen wurde (vgl. auch die in 4.2 dargestellte glaubwürdige Einbindung in den politischen Prozess). Dies ist allerdings schwer zu kommunizieren und damit zu beurteilen. Ein Indiz hierfür kann sein, dass auch zu anderen Gelegenheiten bereits nicht vorgeschriebene Formen der Bürgerbeteiligung angewendet wurden. Grundsätzlich besteht die Gefahr, dass es den Veranstaltern mehr um Profilierung (als „innovativ“ und „,neuen Technologien gegenüber aufgeschlossen“) geht denn um die Meinungen und Kenntnisse der Bürgerinnen und Bürger.

Zur kulturellen Anschlussfähigkeit gehört auch die Frage der anonymen Nutzung dieses neuen Mediums. In der politischen Diskussion innerhalb einer offenen Demokratie sollte der Grundsatz der Authentizität herrschen, d. h. dass alle Beteiligten zu ihrer Position stehen. Staatsbürgerschaftliches Denken und Verhalten werden durch anonyme Beteiligung nicht herausgefordert. ${ }^{15}$ Es ist unklar, wer und wie viele Bürger sich wirklich beteiligen. „Durch die Möglichkeit anonymer Beiträge wird das Meinungsbild verzerrt," so ein Politiker in der Forumsauswertung. Zu seiner Meinung stehen zu können bedeutet auch, Verantwortung zu übernehmen. Anonymität kann zwar als Ventil für u. U. (sozial) unerwünschte Meinungsäußerungen genutzt werden, sollte aber in diskursiven Pha-

14 Vgl. die E.D.E.N.-Webseite www.edentool.org und hier insbesondere das Deliverable D2.1, in dem die Nutzeranforderungen (User Requirements) erhoben wurden.

15 Ein Bürger formulierte diesen oft vertretenen Standpunkt in der Begleituntersuchung so: „Die anonymen Meinungen finde ich befremdlich; wer sich hier beteiligt, sollte dies mit „offenem Visier“ tun.“ 
sen die Ausnahme bleiben, weil es vielfach unproduktives „Gemeckere“ fördert und zum unbewussten Abschieben von eigener Verantwortung auf andere führen kann. Für einen anonymen (neben authentifiziertem) Zugang sprechen allerdings auch gute Gründe: ${ }^{16}$

- Nur eine Minderheit traut sich bei traditionellen Verfahren, den „Mund auf zu machen”. Es gehören Mut und Ausdrucksvermögen dazu, in Versammlungen leibhaftig (gestikulierend und mit Körpersprache) oder in Leserbriefen mit eigenem Namen zum eigenen Standpunkt zu stehen.

- Aufgrund der Anonymität trauen sich auch Bürgerinnen und Bürger ihre Meinung zu sagen, die weniger sprachgewandt sind und es sonst nicht tun würden (vgl. Westholm 2002a). Dieses Argument gilt auch für bestimmte Inhalte; bei manchen Themen führt erst die Möglichkeit der anonymen Äußerung dazu, dass die eigene Meinung oder Erfahrung überhaupt artikuliert wird und Meinungen bekannt werden, die die Verwaltung oder Politik sonst so nicht erfahren würde. Dies hat das britische Parlament bei einer Anhörung zum Thema häusliche Gewalt erfahren.

- Diese Positionen sind bei jedem erneuten Versuch sorgfältig gegeneinander abzuwägen. Vor dem Hintergrund, dass alle User noch Erfahrungen mit dem neuen Medium machen sollten, überwiegen derzeit noch die Argumente für Anonymität.

\subsection{Die Rolle der Politik}

Die fünf genannten Ebenen werden allesamt von der politischen Anschlussfähigkeit und den Handlungen politischer Entscheidungsträger beeinflusst, die ihrerseits dem Druck von wirtschaftlichen und anderen Interessengruppen unterliegen: Die Politik entscheidet in ihrem Streben nach Legitimation und Mehrheiten über Gesetzesänderungen z. B. zur Stärkung von Beteiligungsrechten wie auch über die Mittelbereitstellung und damit über Richtung und Tempo der eDemocracy-Entwicklung. Daher kommt der politischen Anschlussfähigkeit eine Schlüsselrolle zu. Es stellt sich die Frage, was Politik und Verwaltung dazu motivieren sollte, den Bürgern freiwillig größere Mitwirkungsmöglichkeiten einzuräumen und damit - vermeintlich - die eigene Position zu schwächen. Dies muss allerdings keineswegs das Ergebnis von unmittelbarer Beteiligung sein. Vielmehr gibt es auch Beispiele für (mittelbare) Konfliktlösungen und Konsensbildung über Stellvertreter auf diesem Weg, die Politik und Verwaltung dann als Erfolg verbuchen können. Ob mit oder ohne Internet - die Spielregeln der politischen Entscheidungsfindung berühren letztlich immer auch Machtinteressen. Indem Politik und Verwaltungsspitze selbst Veränderungsbereitschaft zeigen und sich zum obersten Fürsprecher für mehr Bürgerbeteiligung machen („Leadership“), können die weiteren notwendigen Änderungen im Verwaltungssystem angestoßen werden. In dem Fallbeispiel war es ein besonders motivierter Akteur in der (Ortsamts-) Verwaltung, der eigene Verbindungen ausnutzte, um eine kostengünstige Realisierung dieser innovativen Anwendung zu ermöglichen. Die Politik spielte eher die Rolle der „Bedenkenträger“, wobei nicht darüber geurteilt werden soll, ob dafür das vereinzelt geäußerte Argument der vermeintlich geringen Effektivität der ausschlaggebende Faktor war oder die (nicht artikulierte) Angst vor Abgabe von Einflussmöglichkeiten.

Finanziell sollten vor dem Hintergrund von Einsparungen in Kommunalverwaltungen attraktivere Lösungen gefunden werden; isoliert stehende Foren sind teuer in der Anschaffung und im Betrieb. Aus diesem Grund bieten sich Application Service Provider-Lösungen an, in denen ein Dritter (z. B. ein kommerzieller Betreiber) Beteiligungstools anbietet, die die Kommune nutzen und an ihr System und ihre Corporate Identity einpassen kann (vgl. Kubicek/Westholm/Wind 2002).

16 Zur ausführlicheren Diskussion über Anonymität bei Online-Foren vgl. Westholm 2002b. 
Wenn man die Chancen von Angeboten für Online-Bürgerbeteiligung beurteilen will, muss die Anschlussfähigkeit in allen aufgezeigten Feldern gleichermaßen geprüft werden. Was als relevanter Kontext zu berücksichtigen ist, entscheidet sich im konkreten Einzelfall. Die Anschlussfähigkeit ein und derselben Anwendung kann in der einen Stadt unproblematisch sein, aber schon in der Nachbargemeinde völlig entgegengesetzt beurteilt werden. Dabei ist zu berücksichtigen, dass wir es nicht mit statischen, sondern veränderbaren Systemen zu tun haben: Zeigt sich eine Kommune als besonders innovativ und hat damit Erfolg, werden sich andere dieser Entwicklung nicht dauerhaft verschließen können.

\section{Zusammenfassung und Ausblick}

Neue Medien in Form von eDemocracy können deliberative Politik stützen v. a. dadurch, dass sie zu verbesserter Informiertheit beitragen. Der Vorteil der Asynchronität, also die teilweise Unabhängigkeit von Zeit und Ort, ermöglicht, dass das Internet primär zur Entscheidungsvorbereitung genutzt wird, die Bürger kommen informierter zu Gesprächen in die Verwaltung (bzw. sie können sich einen Weg sparen). Dagegen ist das Internet (noch) nicht geeignet, bislang nicht interessierte Bevölkerungskreise für Planungsprozesse zu gewinnen, sondern es spricht (bislang) meist privilegierte Bevölkerungskreise an und stärkt v. a. diejenigen, die politische Kommunikation gewohnt sind - soziale Unterschiede, die bei herkömmlichen Beteiligungsformen bestehen („Standardmodell der Beteiligung“), werden vom „Digital Divide“ überlagert und teilweise verschärft. Die Möglichkeit der anonym(isiert)en Nutzung des Mediums stellt eine Herausforderung dar insofern, als eine breitere Basis für Partizipation erreicht werden könnte, indem sich Teilöffentlichkeiten mischen. Andererseits geht Authentizität verloren, so dass eine von Zivilcourage getragene Staatsbürgerrolle nicht unbedingt Unterstützung erfährt.

Online-Foren sowohl in der politisch unverbindlicheren Form der Konsultation als auch in stärker verbindlicher Form der Mediation binden finanzielle und personelle Ressourcen. Die „Bürgermoderation“ in Horn-Lehe hat hier einerseits Ansätze gezeigt, bürgerschaftliche Ressourcen zu nutzen, andererseits aber auch ehrenamtliches Engagement von Bürgern gezielt zu fördern. Bei OnlineForen müssen zudem Möglichkeiten des Content-Management gefunden werden, quasi Filter, die die Beiträge für bestimmte Zielgruppen (Bauverwaltung und -politik, Jugendverwaltung und -politik etc.) selektieren und ggf. aufbereiten, damit die vielen Informationen an die richtigen Adressaten gelangen. „Content-Management'“ bezieht sich ebenso auf die Frage, wie Hintergrundinformationen nutzungsgerecht präsentiert werden. Unklar ist, ob dieses Argument der begrenzten „Informationsverarbeitungskapazität" des politisch-administrativen Systems nicht vorgeschoben wird und als Ausrede dient, um geringes Interesse an Bürgermitwirkung zu verschleiern.

Perspektivisch werden die größten Potentiale der IuK (neben der Informationsvermittlung) in mediationsähnlichen Verfahren gesehen, die durch die neuen Medien zwar nicht ersetzt, aber sinnvoll effektiviert werden können. Aufgrund des gesellschaftlichen Wandels weg von hoheitlicher Regulierung hin zu kooperativen Verfahren (Governance-Strukturen), bei denen auch Gruppen des dritten Sektors in die Entscheidungsfindung einbezogen werden, verspricht man sich eine stärkere Einbindung solcher Verfahren in Entscheidungsverfahren (vgl. PRISMA 2002) ${ }^{17}$.

17 Im EU-Projekt PRISMA (Providing Innovative Service Models and Assessment) wurden eGovernment-Strukturen in unterschiedlichen Servicefeldern (Verwaltung, Gesundheit, Umwelt u. a.) untersucht und Szenarien für Entwicklungen in den nächsten zehn Jahren entwickelt. 
Online-Foren (als Konsultation wie Mediation) haben den Vorteil, dass sie asynchron genutzt werden können. Andererseits entgeht ihnen das Lebendige von Bürgerversammlungen, in denen persönliche Anwesenheit und Authentizität gefragt sind. Für zwischenmenschliche Kommunikation wichtige Faktoren wie Mimik, Gestik und Stimmlage drohen im neuen Medium völlig unterzugehen. Dies bedeutet auch eine Verarmung politischer Kommunikation. Dagegen stehen ansatzweise gute Erfahrungen im Horn-Leher Forum mit Responsivität und Empathie unter den Diskutanten.

Grundsätzlich sollte hieraus gefolgert werden, einen Multi-channel-Ansatz zu verfolgen, d. h. unterschiedliche Bürgerbeteiligungsverfahren zu kombinieren, um die jeweiligen Stärken der unterschiedlichen Verfahren zur Geltung zu bringen. Förderung innovativer Technologien als Selbstzweck reicht als Argument nicht aus in Zeiten, da härter um (finanzielle) Ressourcen der öffentlichen Hand, und insbesondere der Kommunen, gerungen werden muss. Es bedarf aber noch weiterer Modellversuche, in die vorhandene Erfahrungen einfließen können; die Berücksichtigung der verschiedenen Dimensionen von Anschlussfähigkeit ist eine Grundvoraussetzung.

\section{Literatur}

Bischoff, Ariane/Selle, Klaus/Sinning, Heidi (1996): Informieren, Beteiligen, Kooperieren. 2. Aufl. Dortmund.

Coleman, Stephen/Götze, John (2002): Bowling Together: Online Public Engagement in Policy Deliberation. London.

Dalton, Russel J. (1988): Citizen Politics in Western Democracies. Public Opinion and Political Parties in the United States, Great Britain, West Germany, and France. Chatham N.J.

Fisahn, Andreas (1996): Kampf gegen Windmühlen? Überlegungen zur Bedeutung der Bürgerbeteiligung für den Umweltschutz anlässlich der Beschleunigungsnovellen. In: Neue Justiz, H. 2, S. $63-70$.

Frowerk, Jens-Ulrich/Sombetzki, Christian/Stiftung Mitarbeit (Hrsg.) (1999): Kinderpolitik - Kinderbeteiligung. Kinder- und jugendpolitische Beteiligungsmodelle. Bonn.

Habermas, Jürgen (1997): Drei normative Modelle der Demokratie. In: Ders.: Die Einbeziehung des Anderen. Studien zur politischen Theorie. Frankfurt/M., S. 277-292.

Hohn, Susanne (2001): Bürgerbefragung zum Thema: Das Internet als Instrument der Bürgerbeteiligung, untersucht am Beispiel des Flächennutzungsplanes der Stadt Osnabrück. 2. MEDIA@Komm-Kongress, Esslingen, 12./13.6.2001 (mimeo).

Holtkamp, Lars. (2000): Bürgerbeteiligung in Städten und Gemeinden. Ein Praxisleitfaden für die Bürgerkommune. (Hg.: Heinrich-Böll-Stiftung). Berlin.

KGSt (1999): Bürgerengagement - Chance für die Kommunen. KGSt-Bericht 6/99. Köln.

Kubicek, Herbert/Westholm, Hilmar (2002): Selbstzweck oder Notwendigkeit: Wie kann E-democracy Bürgerbeteiligung erweitern? In: eGov Präsenz. Fachzeitschrift des Kompetenzzentrums eGovernment, Bern, S. 4-8.

Kubicek, Herbert/Westholm, Hilmar/Wind, Martin (2002): Wahlen und Bürgerbeteiligung via Internet. In: A. Meier (Hg.): E-Government (zugleich HMD Praxis der Wirtschaftsinformatik H. 226), Heidelberg, S. 21-36. 
Leggewie, Claus/Bieber, Christoph (2001): Interaktive Demokratie. Politische Online-Kommunikation und digitale Politikprozesse. In: Aus Politik und Zeitgeschichte. B 41-42/2001, S. 37-45.

Lenk, Klaus (1999): ,Electronic Democracy' - Beteiligung an der kommunalen Willensbildung. In: H. Kubicek u. a. (Hg.): Multimedia@Verwaltung. Jahrbuch Telekommunikation und Gesellschaft 1999, Heidelberg, S. 248-256.

Luhmann, N., 1975, Legitimation durch Verfahren, Frankfurt/M.

OECD (2001): Engaging Citizens in Policy-Making: Information, Consultation and Public Participation. PUMA Policy Brief No. 10; http://www.oecd.org/pdf/M00007000/M00007815.pdf (Download: 3.11.2002).

PRISMA (2002): Pan-European Best Practice Models. Deliverable 5.1 within the EU-Project PRISMA (Providing Innovative Servise Models and Assessment). (www.prisma-eu.net).

Schneider, F., 2001, Demokratie goes Online. Internet-basierte Bürgerbeteiligung in der Bauleitplanung. Chancen und Grenzen aus der Sicht der Planungspraxis. 2. MEDIA@KommKongress, Esslingen, 12./13.6.2001.

Schwabe, G./Krcmar, Helmut (1999): Cuparla - Telekommunikation im Stuttgarter Kommunalparlament. In: Scheer, A.-W./Nüttgens, M. (Hg.): Electronic Business Engineering. Heidelberg.

Trénel, Matthias/Märker, Oliver/Hagedorn, Hans (2001): Bürgerbeteiligung im Internet - Das Esslinger Fallbeispiel. (Reihe: WZB-paper FS II 01-308). Berlin.

Westholm, Hilmar (2002a): (Mehr) Partizipation über Internet? Fallbeispiel einer Online-Meinungsumfrage. In: Rundbrief Bürgerbeteiligung I, hg. von der Stiftung Mitarbeit, S. 4-9.

Westholm, Hilmar (2002b): Neue Medien für bessere Bürgerbeteiligung in der „Bürgerkommune“? Ein Praxisbericht. In: Prigge, R../Osthorst, W. (Hrsg.): Bremen auf dem Weg zur Bürgerkommune? Bremen (im Erscheinen).

Zilleßen, Horst (1998): Mediation. Kooperatives Konfliktmanagement in der Umweltpolitik. Opladen 1998. 


\section{Bisher erschienene manu:scripte}

ITA-0I-0I Gunther Tichy, Walter Peissl (12/2001): Beeinträchtigung der Privatsphäre in der Informationsgesellschaft. <http:/www.oeaw.ac.at/ita/pdf/ita_01_01.pdf>

ITA-0I-02 Georg Aichholzer(12/2001): Delphi Austria: An Example of Tailoring Foresight to the Needs of a Small Country. <http://www.oeaw.ac.at/ita/pdf/ita_01_02.pdf>

ITA-0I-03 Helge Torgersen, Jürgen Hampel (12/2001): The Gate-Resonance Model: The Interface of Policy, Media and the Public in Technology Conflicts.

$<$ http://www.oeaw.ac.at/ita/pdf/ita_01_03.pdf >

ITA-02-0I Georg Aichholzer (01/2002): Das ExpertInnen-Delphi: Methodische Grundlagen und Anwendungsfeld ,Technology Foresight“.

$<$ http://www.oeaw.ac.at/ita/pdf/ita_02_01.pdf>

ITA-02-02 Walter Peissl (01/2002): Surveillance and Security - A Dodgy Relationship. $<$ http://www.oeaw.ac.at/ita/pdf/ita_02_02.pdf>

ITA-02-03 Gunther Tichy (02/2002): Informationsgesellschaft und flexiblere Arbeitsmärkte. $<$ http://www.oeaw.ac.at/ita/pdf/ita_02_03.pdf $>$

ITA-02-04 Andreas Diekmann (06/2002): Diagnose von Fehlerquellen und methodische Qualität in der sozialwissenschaftlichen Forschung.

$<$ http://www.oeaw.ac.at/ita/pdf/ita_02_04.pdf>

ITA-02-05 Gunther Tichy (10/2002): Over-optimism Among Experts in Assessment and Foresight. $<$ http://www.oeaw.ac.at/ita/pdf/ita_02_05.pdf $>$

ITA-02-06 Hilmar Westholm (12/2002): Mit eDemocracy zu deliberativer Politik? Zur Praxis und Anschlussfähigkeit eines neuen Mediums.

$<$ http://www.oeaw.ac.at/ita/pdf/ita_02_06.pdf $>$ 